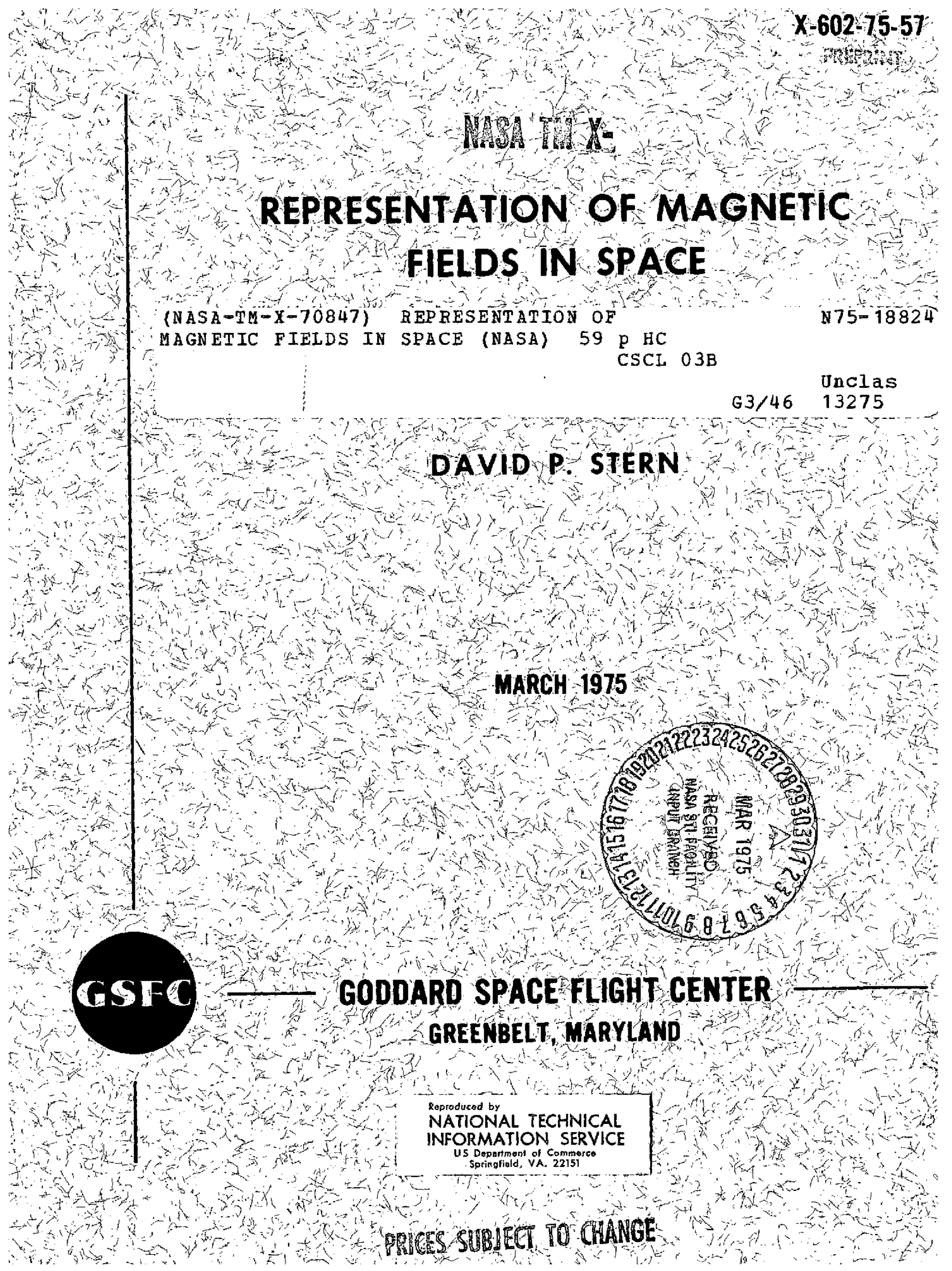




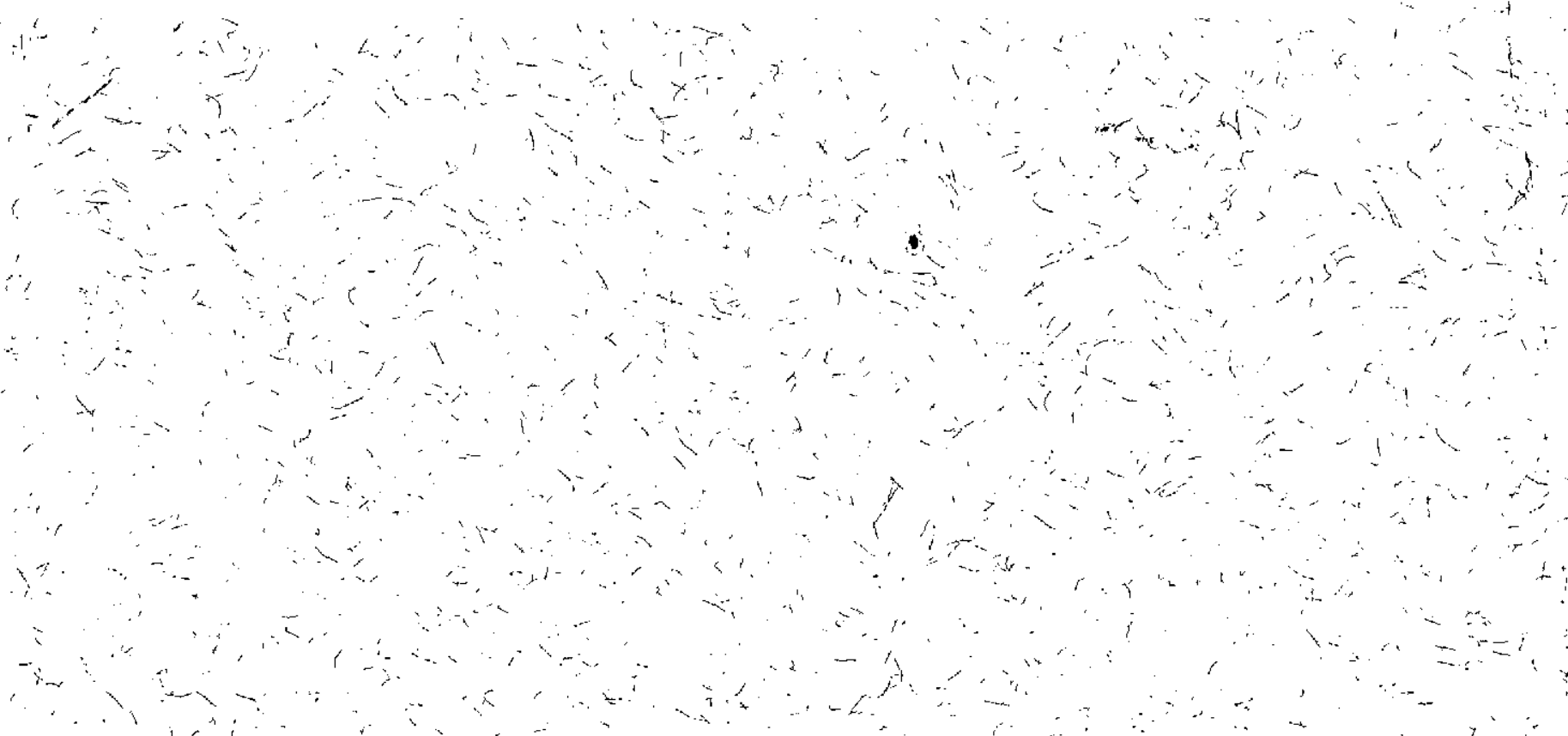

For information concerning availability of this document contact:

Technical Information Division, Code 250 Goddard Space Flight. Center Greenbelt, Maryland 20771

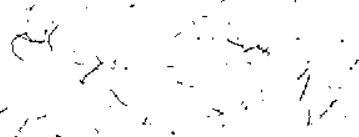

(Telephone $301-982-4488$ )

"This paper preserits the views of the author(s), and does not nocessarly reflect the views of the Goddard Spece Flight Center; or NASA." 


\title{
Representation of Magnetic \\ Fields in space
}

\author{
David $P$ 。 Stern \\ Theoretical Studies Group \\ Goddard Space Flight Center \\ Greenbelt, Maryland 2077I.
}

To be submitted to Reviews of Geophysics and Space Physics 


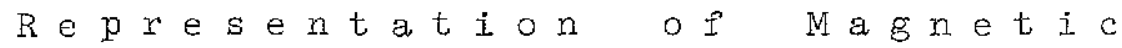

$$
\text { Fie } 1 \mathrm{~d} s \text { in } \mathrm{s} p \mathrm{p} \in \mathrm{e}
$$

IINPRODUCTION

(I) THE VECTOR POPENTIAL A

(2) THE SCALAR POTENTIAL $\gamma$
(a) Introduction
(b) Spherical Harmonics
(c) Geomagnetic Models
(d) Current-iree Models of the Magnetosphere
(e) Change of Coordinates
(i) Practical Problems related to Uniqueness

(3) TOROIDAL AND POLOIDAL FIELDS
(a) Introduction
(b) Vector Potential for Curl-free Fields
(c) Models of the Magnetosphere
(a) Spherical Vector Harmonics

(4) EULER POTENTIALS

(a) Definition and General Properties

(b) Examples

(c) The I Parameter.

(5) LOCAL REPRESENTATIONS

AFTERWORD 


\section{INPRODUCTION}

The purpose oI this work is to review several methods by which a magnetic iield $\underline{B}$ in space can be represented, with particular attention to problems of the observed geomagnetic field.Time dependence will be assumed to be negligible and five main classes o $\vec{I}^{\circ}$ representation will be described, as follows:

(1) Representation by a vector potential A :

$$
\underline{B}=\nabla \times \underline{A}
$$

This is a general form to which other Iorms noted here can always be converted.

(2) Representation by a scalar potential $\gamma$ :

$$
\underline{B}=-\nabla y
$$

This representation is available. only in current-Iree regions and it is particularly appropriate to the main geomagnetic ileld, where the expansion of $\gamma$ in spherical harmonics is of considerable interest.

(3) Representation by orthogonal vectors, in particular those related to spherical coordinates

$$
\underline{B}=\nabla \times \Psi_{1} \underline{r}+\nabla \times \nabla \times \psi_{2}^{\prime} \underline{r}
$$

This representation is related to spherical vector harmonics; it has been used in dynamo theory and in a variety of problems. As will be seen, it is also useful in devising models of the earth's magnetosphere iield. 
(4) Representation by Euler potentials

$$
\underline{B}=\nabla \alpha \times \nabla \beta
$$

This is the only representation which includes explicit information about the configuration of magnetic field lines, but its nonlinear character makes its derivation dificicult. In the earth's magnetic field $\alpha$ is related to McIlwain's I parameter which is useful in the study of the motion of trapped particles.

(5) Local representations, in which B is expanded around its value at some reference point

$$
\underline{B}=B_{0}+\underline{r} \cdot \nabla B_{0}+\underline{\underline{l}} \underline{\underline{r}} \underline{r}: \nabla \nabla B_{0}+\ldots
$$

Such expansions Iind use in the theory of guiding center motion and in describing the vicinity or neutral points at which B vanishes.

The present discussion is not intended to be self-contained: matters on which recent reviews exist in the general literature will only be briefly described with reierences directing the reader to more elaborate treatments. Reierences will also be given to articles in which various mathematical tools described here are employed or mentioned, but the mentioning of such articles does not always imply concurrence with all conclusions expressed there, nor is the review of the literature meant to be complete. In some cases review papers are cited mainly in order that they may provide the reader with a guide to earlier work not mentioned here. 


\section{(1) THE VECTOR POTENTIAI A}

The representation (1) is the most general one and others may be reduced to it. For instance, (3) leads naturally to

$$
\underline{A}=\psi_{I} \underline{\underline{r}}+\nabla \times \psi_{2} \underline{r}
$$

while (4) gives

$$
\underline{A}=\alpha \nabla \beta
$$

which is orthogonal to $\mathrm{B}$. The representation (2) also has an equivalent form (1) and if $\gamma$ is expanded in spherical harmonics an equivalent representation of the Iorm (3) is readily obtained (as will be shown), allowing (6) to be used. All these choices of $\underline{A}$ are indeterminate within the gradient of an arbitrary scalar $\psi_{0}$, since the addition or such a gradient to $\underline{A}$ does not aîlect (1).

The representation (1) is part of the standard treatment found in practically all texts on classical electrodynamics and will therefore not be discussed in detail. Its main usefulness, in that $\mathbf{I}$ orm, is that î the current density $\underline{j}$ creating the field is everywhere known, A is readily derived (assuming $B$ to be a vacuum field) by volume integration

$$
\underline{A}(\underline{r})=\left(\mu_{0} / 4 \pi\right) \int \frac{\underline{j}\left(\underline{r}^{2}\right)}{\left|\underline{r}-\underline{r}^{\top}\right|} d V^{\prime}
$$

In engineering applications $\underline{j}$ is often given by the circuit geometry and $\underline{A}$ is readily calculated (orten, $\underline{B}$ is directly derived in such cases from the Biot-Savart iormula). In space, on the other hand, $\underline{\perp}$ is generally a derived quantity obtained only arter $\underline{B}$ is given, often with low accuracy, so that (8) is used relatively infrequently.

One important case in space science where $j$ is given occurs in regions where it is inferred from the observed Iluxes of charged particles 
[e.g. Northrop, 1963, eq. 4.1 ; Longmire, 1963]. One such region occurs in the vicinity of the earth, where trapped particles support a current density often called (not entirely accurately) the ring current. The magnetic field in these regions is generally known from independent observations and its comparison to $\underline{j}$ forms an interesting check, often enabling one to deduce which part of an observed particle population contributes most to the magnetic perturbation. Many analyses of the ring current obtain $\underline{B}$ directly from $\underline{j}$ via the Biot-Savart formula [e.g. Akasofu and Chapman, 1961; Schield, 1969 ]. However, general formulas for A, derived by (8) for configurations used in calculations of the erfects or a ring current, have been published by Kendall et al. [1966].

Another case in which distributions of current density are used as the source of magnetic fields in space occurs when such distributions are introduced as the input data of theoretical models. The configuration of the current in such cases may be selected on physical grounds - e.g. Field-aligned currents [ Bonnevier et al., 1270; Crooker and Siscoe, 1974] or the geomagnetic tail sheet current [Williams and Mead, 1965] or else it (or part of it) may be represented by a generally expanded function of position. The advantage of using $j$ in the latter case is that this assures the vanishing of $\boldsymbol{\nabla} \cdot \underline{B}$, which may have been the reasoning of 01son [1974; 01son and Pfitzer, 1974]. Later on in this review, however, it will be shown that similar properties may be obtained by simpler means. In most such applications, the Biot-Savart formula is used and $B$ is derived directly. 


\section{(2) THE SCAIAR POITENIAL $\gamma$}

(a) INTRODUCTION

In current-Iree regions $\nabla \times \underline{B}$ vanishes and it is possible to represent $\underline{B}$ by a scalar potential

$$
\underline{B}=-\nabla \gamma
$$

Because $\nabla \cdot \underline{B}=0, \gamma$ is harmonic

$$
\nabla^{2} \gamma=0
$$

and may be expanded in a variety of ways appropriate for harmonic functions. The properties of harmonic functions and of $\gamma$ in particular are discussed in most texts of classical electrodynamics and for this reason the discussion here will be confined to applications relevant to the geomagnetic field.

(b) SPHERICAL HARMONICS

The "main" geomagnetic field - i.e. that part of the field observed at or above the earth's surface which is caused by currents in the earth's interior - accounts for about $99 \%$ of the field observed at ground level and is readily expanded in extemal spherical harmonic functions

$$
\gamma=a \sum_{n=1} \sum_{m=0}^{m=n}(a / r)^{n+1} P_{n}^{m}(\theta)\left(g_{n}^{m} \cos m \varphi+h_{n}^{m} \sin m \varphi\right)
$$

where a is the earth's radius and $P_{n}^{m}$ are associated Legendre functions. Attention should be given to the factor a outside the surmation, intended to give the coefficients $\mathbb{E}_{n}^{m}$ and ${ }_{h}^{m}$ the dimensions of magnetic 
field : as a consequence such coeficients are generally given in units of gausses or gammas (one gamma $=1 \gamma=10^{-5}$ gauss). The form given in (11), involving real coeficients, is preferred in geomagnetic research over the one used in mathematical treatments, where the last factor in each term or (11) is expressed in terms of $\psi_{n}^{m} \exp$ (im $\varphi$ ), with the summation over $m$ extenaing from $-n$ to $n$ and the coefficients $\left(\psi_{\mathrm{n}}^{\mathrm{m}}, \psi_{\mathrm{n}}^{-\mathrm{m}}\right.$ ) being complex conjugates ( a related representation uses spherical harmonic functions $Y_{n}^{m}(\theta, \varphi)$ proportional to $P_{n}^{m}(\theta) \exp \operatorname{im} \psi$ ). Properties and details of this expansion are reviewed in many texts and articles $[\mathrm{e} . \mathrm{g}$. Chapman and Barte1s, 1940; Heppner, 1963 ; Kauld , 1968; Stacey, 1969; Zmuda, 1973] 。

In using the spherical harmonic expansion of $\gamma$ note should be taken of the choice of normalization, for several deinitions of legendre functions, diriering by constant factors, are currently employed.

Three main choices of this kind are described by Chapman and Bartels [1240]; in the notation used by them (adopted from Adolf Schmidt) Legendre Iunctions are denoted $P_{n, m}$ for mathematical normalization, $\mathrm{P}^{\mathrm{n}, \mathrm{m}}$ Ior gaussian normalization and $\mathrm{P}_{\mathrm{n}}^{\mathrm{m}}$ Ior Schmidt normalization. The same notation will be used here but it is by no means a general one and frequently authors denote Legendre Iunctions by $P_{n}^{m}$ regardiless of the normalization which they are using.

In the above notation, if $\mu=\cos \theta$

$$
\begin{aligned}
P_{n, 0}(\mu)= & \left((-1)^{n} / 2^{n} n !\right) a^{n} / a \mu^{n}\left(1-\mu^{2}\right)^{n} \\
& \text { (Iegendre polynomials) } \\
P_{n, m}(\mu)=\left(1-\mu^{2}\right)^{m / 2} a^{m} P_{n, 0} / a^{m} & \\
P^{n, m}(\mu)= & {[(n-m) ! / 1.3 \cdot \ldots(2 n-1)] P_{n, m}(\mu) } \\
& =\left[(n-m) ! n ! 2^{n} /(2 n) !\right] P_{n, m}(\mu)
\end{aligned}
$$




$$
\begin{aligned}
& P_{n}^{O}=P_{n, 0} \\
& P_{n}^{m}=[2(n-m) ! /(n+m) !]^{1 / 2} P_{n, m}(m \neq 0) \\
& P_{n}^{O}=[1 \cdot 3 \cdot 5 \cdot \ldots \cdot(2 n-1) / n !] P^{n, 0} \\
& P_{n}^{m}=\left\{1 \cdot 3 \cdot 5 \cdot \ldots \cdot(2 n-1) /\left[\frac{1}{2}(n+m) !(n-m) !\right]^{1 / 2}\right\} P_{n}^{m}
\end{aligned}
$$

The coefficients of (11) gencrally have their indices arrayed in the same manner as the Iegendre runctions to which they are attached. In transiorming (11) Irom one normalization to another, whenever a Legendre Iunction is multiplied by some factor, the corresponding harmonic coefricient has to be divided by the same factor, ensuring that $\gamma$ does not vary.

In the literature coefficients of the expansion of $\gamma$ are generally listed for either Schmidt or gaussian normalization: for easy identification the convention exists to reverse all coefficient signs for gaussian normalization, so that the axial dipole coeficient $B_{I}^{0}$ is negative for Schmiat-normalized potentials but positive for gaussian ones. In Schmiat normalization the magnitude of terms is roughly of the order of their contribution to the field: their gradual decrease with growing $\mathrm{n}$ reflects the relative preponderance (near the earth's surface) of the contribution of low-order harmonics, and for any $n$ they do not vary systematically with $\mathrm{m}$.

In gaussian normalization the magnitude of terms decreases more gradually with $\mathrm{n}$ and ror any $\mathrm{n}$ the terms having low $m$ tend to be larger. In computer applications the derivation of $\mathrm{P}_{\mathrm{n}, \mathrm{m}}$ is, however, 
much simpler than that of $P_{n}^{m}$, because it can be handled by means of a recursion relation iree from any irrational factors, connecting terms with the same m [Cain et al. , 1967 ]:

$$
\begin{aligned}
& P_{m, m}(\theta)=\sin ^{m} \theta \\
& P_{m+1, m}(\theta)=\cos \theta \sin ^{m} \theta \\
& P_{n+1, m}(\theta)=\cos \theta P_{n, m}+\frac{(n+m)(n-m)}{(2 n+1)(2 n-1)} P_{n-1, m}
\end{aligned}
$$

The derivatives required for the calculation of $\mathrm{B}_{\theta}$ are best found by a recursion relation based on the derivatives of the above relations, starting with

$$
d P_{m, m} / d \theta=m \cos \theta \sin ^{m-1} \theta
$$

Normalizations other than those described also exist: a comparative list of 8 choices from 18 choices has been compiled by Kaula [1965; Table 1].

(c) GEOMAGNETIC MODEILS

Expansions of the form (11) have been used for the geomagnetic field since the time of Gauss [1839] - Typically, model expansions use harmonics with $n$ ranging up to $\mathrm{n}_{\max }$, with $\mathrm{n}_{\max }$ between 6 and 10 . Since the number of independent coefficients in such an expansion is $\left(n_{\max }+1\right)^{2}-1$, one often iinas authors speaking about models with $48,63,80,99$ or 120 coefficients. 
A very comprehensive review of early analyses of the main field has been compiled by McDonald and Gunst [1967] and some early results are also given by Chapman and Bartels $[\underline{1940}]$. More recent field models have been listed by Heppner [1963] (gaussian normalization) and by Vestine [1960] (Schmidt-normalized) - Cain [1971] reviewed some other recent work and analyzed problems which arise in connection with field mapping by satellite. An International Geomagnetic Rererence Field IGRF 1965.0 was derived and published by IAGA Commission 2, Working Group 4 [1969] and contains 80 terms.

Most of these models taike into account the slow "secular" variation of the field by assuming a linear dependence oi the coerificients $\left(g_{n}^{m}, h_{n}^{m}\right)$ of the form

$$
a=a_{0}+\dot{a} t
$$

where $a_{0}$ is the value of the coeficient at some initial time (e.g. the beginning of 1965 Ior the IGRF model mentioned belore) and $t$ is in years. Some models - for example that or Cain et al. [1967]-also include correction terns proportional to $t^{2}$.

\section{(d) CURRENT-FREE MODELS OF THE MAGNETOSPHERE}

The expansion (1工) consists solely of "intermal harmonics" representing a field which originates inside the earth and vanishes at infinity. I $\vec{r}$ sources external to the earth were also included their contribution to $\gamma$ could be represented by a series of "external harmonics" with positive powers of $r$

$$
\gamma^{\prime}=a \sum_{n=1} \sum_{m=0}^{m=n}(r / a)^{n} P_{n}^{m}(\theta)\left[\bar{E}_{n}^{m} \cos m \varphi+\bar{h}_{n}^{m} \sin m \varphi\right]
$$

Models of this Iorm have been proposed for the magnetospheric rield by Mead $[1964]$ and by Midgeley $[1964]$. In both cases the earth's 
field was approximated by the axial dipole given by $B_{2}^{0}$ in (11), which is orthogonal to the earth-sun line, and the Irame of coordinates for both the dipole component and the expansion (20) is fixed with respect to the sun and the plane of the ecliptic.

In more complicated models internal and external ìields are usually expressed in different frames of reierence, since the internal Iield alone co-rotates with the earth. If one neglects all internal harmonics except Ior the three dipole coefficients $g_{1}^{0}, g_{1}^{1}$ and $h_{1}^{1}$, the model will depend (when external conditions are iixed) only on one parameter which varies in time - the angle between the earth's dipole axis and the eart-sun line, which varies by about $\pm 35^{\circ}$ around $90^{\circ}$, depending on the season of the year and the rotation of the carth. For any given value of this angle such a model is symmetrical about the plane containing the dipole axis and the earth-sun line and it is conveniently described in solar geomagnetic coordinates [01son, 1969 (where the caption of Fig. I should be ignored); Russel1,1971; Burch and Janetzke, 1974] with the origin at the earth's center, the $x$-axis pointing sunward, the $x-z$ plane containing the dipole axis and the z-axis pointing into the northern hemisphere.

Current-iree models of this kind are not meant to represent exterm nal ifielas observed on the earth's surface - indeed, the suriace efiects predicted by such models are far too small to account Ior the observed daily variation [Mead, 1964] . Instead, they are intended to approximate the large-scale field of the outer magnetosphere. In a qualitative way they seem to agree with observations - in particular, they display a sharp boundary on the sunward side, limiting the region of field lines connected to the earth and corresponding to the observed magnetopause. The most signiricant non-dipole coefilieients seem to be $\bar{g}_{1}^{0}$ and $\bar{g} \frac{1}{2}$ and Iurther improvement has been obtained [Williams and Mead, 1965] by adding a current sheet across the median plane of the geomagnetic tail. 
Another simple current-free model approximating the external magnetosphere is the image dipole model; here the effects of the external rield are approximated by a dipole parallel to the z-axis but located some distance sunward from the earth [Hones, 1963; Taylor and Hones, 1965; Forbes and Speiser, 1971]. This representation, which was inspired by the theory of the magnetic storm developed by Chapman and Ferraro [1931], has two adjustable parameters - the distance to the "image dipole" and its magnetic moment, which generally exceeds that of the earth by a considerable factor. For instance, in the work of Taylor and Hones [1965] the image dipole is 28 times stronger than the axial dipole of the earth (other internal terms are ignored) and is placed 40 earth radii sunward or the earth, at a point which is outside the "magnetopause" and thus beyond the region in which the model is valid; the model also includes a sheet current in the geomagnetic tail region, somewhat similar to the sheet current introduced by Williams and Mead [1965]. In all models with image dipoles the scalar and vector potentials are readily iound by superimposing the contribution of the two dipoles, and it is usually best to leave them in this form and not expand $\gamma$ in spherical harmonics.

\section{(e) CHAIVGE OF COORDIIUTES}

In general the expansion (11) is given in spherical coordinates with the origin at the earth's center and the z-axis aligned with the terrestrial rotation axis. For some applications it is userul to rotate the $\mathrm{z}$ axis so that it coincides with the dipole axis, giving the so-called "dipole coordinates" [ Chapman, 1963] . If $\gamma$ is expanded in dipole coordinates both $B_{i}^{2}$ and $h_{I}^{2}$ vanish and the entire dipole contribution is contained in the axial aipole term $g_{1}^{0}$.

The use of dipole coordinates is especially advisable when it is desired to enhance the preponderance or the axial dipole component over other harmonic terms. For instance, geomagnetic Euler potentials (described later) may be obtained by a perturbation calculation in which 
the zero-order term is the contribution of the dipole rield; it is then clearly advantageous to use dipole coordinates which make this term relatively large and reduce the remaining "perturbation".

Mathematical rormulas exist for transforming (11) Irom geographic to dipole coordinates and they have the virtue that harmonics of a given lower index $n$ contribute in the new coordinates only to harmonic terms with the same $n$. Thus each group of $2 n+1$ coefficients corresponding to a given $n$ transiorms independently and (say) a model with 99 coefricients $(n \leq 9)$ will be represented with full accuracy by the same number oi terms in the new coordinates (to be precise, there will only be 97 independent terms in the new expansion, since $g_{1}^{1}$ and $h_{1}^{1}$ vanish). Formulas Ior such a transformation have been discussed by James ${ }^{1}$ [1969] and are concisely given by Slater [1960].

Where high-speed computers are available it is often simpler to apply a "brute force" transformation as frollows. Suppose a 99-term expansion of $\gamma$ is given: one then begins by deriving its value Irom the given expansion (in geographical coordinates) at 97 points scattered over the surface of the earth. Next one transforms the coordinates of each of the selected points to (spherical) dipole coordinates in which the $\mathrm{z}$ axis is antiparallel to the vector having cartesian components $\left(h_{1}^{1}, g_{1}^{2}, g_{1}^{0}\right)$. In the new coordinates the expansion or $\gamma$ at each of the given points is derived, with the new expansion coefficients $\left(\mathrm{g}_{\mathrm{n}}^{\mathrm{m}^{r}}, \mathrm{~h}_{\mathrm{n}} \mathrm{m}^{\mathrm{r}}\right)$ entering as 97 unknown quantities (two of them vanish and are not counted). This produces 97 equations in y7 unknowns, which are now solved to give the new coefricients. A repetition based on another set of points is recommended as a check on accuracy and as a precaution against ill-conditioned sets of equations, but the resulting set $\overrightarrow{\mid l}$ coefficients is usually accurate enough for normal use. An example of this method is given by Stern [1971] who included in the transformation not only the time derivatives of (19) but also the slow secular variation of the new coordinate axes. 
If the origin of the coordinates is also allowed to vary we obtain eccentric dipole (or "of Iset dipole") coordinates. The choice of an arbitrary origin introduces 3 more adjustable parameters and can be used, for instance, to assure the vanishing not only of $g_{1}^{1}$ and $h_{1}^{1}$ but of $g_{2}^{0}, g_{2}^{1}$ and $h_{2}^{1}$ as well [Bartels, 1936; Chapman and Bartels, 1940] . The $z$ axis of eccentric dipole coordinates should parallel. that of dipole coordinates to assure the vanishing of the two ofi-axis terms with $n=1$.

Uníortunately", a given expansion in geographic or dipole coordinates (e.g. with 99 coefiricients) is no longer accurately represented by the same number of coefiricients (or in general, by any Iinite number) once the origin is shitted. In case of the 80-term IGRF 1965.0 model, Hilton and Schul2 [1973] have shown that a 195-term representation ( $\mathrm{1} \leqslant 13$ ) of the rield in offset dipole coordinates maintains sufficient accuracy Ior practical purposes and have published its coefricients. It may also be noted here that coordinates or the type discussed here seem to be usejul in describing Jupiter's main magnetic Iield, as observed by the Pioneer 10 spacecrait [ Smith et al., 1974] .

\section{(f) PRACTICAI PROBIEMS RETATED TO UNIQUENESS}

The practical derivation of $\gamma$ for the main geomagnetic ileld involves many observational problems, such as the elimination from the data or efiects of external sources and of magnetic anomalies in the earth's crust. Assuming these to be solved, the problem can be viewed as involving the derivation of

$$
\gamma=\gamma_{0}+\gamma_{1}
$$

where $\gamma_{0}$ is some previously known model of the potential and $\gamma_{1}$ is a relatively small correction which is to be derived Irom the given data. If $\gamma_{1}$ is expanded as in (11) in spherical harmonics involving some number $\mathbb{N}$ of unknown coefficients, then any observation related to the 
Iield can be reduced to a linear equation involving these coeficicients. The relation may be exact, e.g. when any component of $\underline{B}$ is observed, or it may be a linearized approximation, as happens when one observes the rield's magnitude $|\underline{B}|$ (see later on) or any of the direction angles detining $\underline{B}$.

Thus the results of a world-wide magnetic survey tend to reduce to a large set of linear equations in $\mathbb{N}$ unknowns. The coefricients of $\gamma_{1}$ are then derived by the least squares procedure as those coming closest to fitting the set and, in case approximate linearization was used in obtaining the equations, $\gamma_{1}$ is generally added to $\gamma_{0}$ to give an improved initial approximation, arter which the procedure is repeated one or more times [Cain et al., 1967$]$.

An interesting problem related to such procedures is that of the uniqueness of the result: how can one be sure that a given data set leads to a unique choice of $\gamma$ ?

One case in which this question has led to unexpected results involves the derivation of $\gamma$ from observations of $|B|$ (commonly denoted in geomagnetism by $F$, a practice which will be followed here). Such observations are easily performed aboard spacecraft with an accuracy or about one gamma [ Cain, 1971 and reierences cited there] and they have the advantage of not requiring precise knowledge of the attitude of the sensor. If one neglects the altitude variation of the orbit, the uniqueness problem reduces to the question whether $\gamma$ is uniquely determined by observation of $|\nabla \gamma|=F$ over the suriace of a sphere.

Linearizing the expression of $F$ obtained Irom (2l) gives

$$
\nabla \gamma_{0} \cdot \nabla \gamma_{1}=\frac{1}{2}\left[\mathrm{~F}^{2}-\left(\nabla \gamma_{0}\right)^{2}\right]
$$

and this can be used iteratively (as described beiore) to derive the coeificients ôे $\gamma$, with $\gamma_{1}$ added to $\gamma_{0}$ after each iteration step to provide a better starting approximation for the step following. In 
computer simulations in which a known expansion (11) was used to generate F this method recovered the input coericients quite accurately within 4-5 steps. Consequently it was generally accepted and widely used in the treatment or actual data.

Meanwhile, however, Backus [1968, 1970, 1974] investigated the problem mathematically. He rirst found that $F$ and $\gamma$ were uniquely related whenever the series (11) was Iinite [Backus, 1968], then he showed by actual counterexamples that this was no longer universally true iI the series (11) was infinite [Bacikus, 1970] and Iinally he proved that this ambiguity was removed if $F$ was observed over a Iinite volume in space

[Backus, 1974].

At the same time evidence began to accumulate suggesting that models derived from $F$ observed in near-circular orbits fit the vector field Iar less accurately than they Iit the distrihution of the field's magnitude. In addition, difterent models derived from $F$ exhibited relatively large differences and this prompted Hurwitz and Knapp [1974] to conduct simulated recoveries similar to those described before but with data contaminated by rinite "noise", as occurs in practical situations. In such cases $\gamma$ is not recovered exactly but finite errors remain and Hurwitz and Knapp found that the fit between the input vector ifield and that derived from the output model was decidedly inferior to the fit between input $F$ and output $F$.

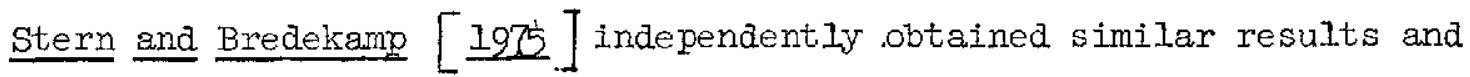
also showed that such effects were connected to the counterexamples of Backus [1970]. These counterexamples bear a special relation to the dipole iield and the ract that the main geomagnetic rield is dominated by its dipole component establishes a connection between them and the problem. In particular, it turns out that in the presence of finite "noise" certain sequences of harmonic terms can exhibit enhanced fluctuations which degrade the itit to vector data much more than they degrade the rit to F. Such enhanced fluctuations were in fact obtained in 
computer simulations and also in analysis of some of the runs of Hurwitz and Knapp [1974] and this strengthens the suspicion that some current models based on $F$ are in lact less accurate than has been claimed.

The uniqueness of $\gamma$ derived Irom some other types or data has been examined by Kono [197] in order to evaluate the correctness of some reconstructions of ancient geomagnetic ilelds. For instance, he proved that magnetic declination observed at the earth's surface does not in general define $\gamma$ uniquely.

(3) TOROTDAI, AND POLOIDAI VECTORS

(a) INRRODUCTION

A general vector rield $\underline{V}$ may be represented by three scalars $\Psi_{O}$, $\psi_{1}$ and $\psi_{2}$, in the form

$$
\underline{V}=\nabla \psi_{0}+\nabla \times \psi_{2} \underline{x}+\nabla \times \nabla \times \psi_{2} \underline{r}
$$

The advantage or this form is (as can be verified by carrying out the algebra) that if $\underline{V}$ satidfies the vector Helmholtz equation

$$
\nabla^{2} \underline{V}+\dot{k}^{2} \underline{V}=0
$$

then each of the $\Psi_{i}$ satisfies the appropriate scalar Helmholtz equation

$$
\nabla^{2} \psi_{i}+k^{2} \psi_{i}=0
$$

(including the case $\mathrm{k}^{2}=0$, when (25) becomes Laplace's equation). In a similar way, if equation (24) is written in cartesian coordinates, it is resolved into three scalar equations of form (25), one for each cartesian 
component of $\underline{V}$; this is use $\hat{I} u l$ if the boundary conditions are easily expressed in cartesian coordinates, whereas (23) bears a similar relation to spherical coordinates. Only a few systems of coordinates allow such direct conversion of the vector equation to the corresponding scalar one [Senior, 1960; Morse and Feshbach, 1953, chapt. 13].

Because (24) arises naturally in wave propagation problems, the representation (23) was Iirst introduced in that context [ Nie, 1908 ; Hansen, $1935]$. Ir a magnetic rield $\underline{B}$ is represented in this manner, $\Psi_{0}$ is generally omitted, since it must then be a harmonic Iunction and its contribution (as will be shown) may be represented by part or $\Psi_{2}$. The representation using this form

$$
\underline{B}=\nabla \times \Psi_{I} \underline{r}+\nabla \times \nabla \times \Psi_{2} \underline{r}
$$

was Iirst introduced in connection with geomagnetic dynamo theory by Elsasser [1945, 1946, 1947, 1956] who called the two components the toroidal and poloidal components of $\underline{B}$, respectively.

These names are still used. To get some intuitive leeling ror their significance, consider axisymmetrical rields, in which neither $\psi_{1}$ nor $\Psi_{2}$ depends on the azimuth angle $\varphi$. A toroidal field oi this type is then aligned with the $\varphi$ direction and has iield lines circling the $\mathrm{z}$ axis, while the Iield lines of a poloidal axisymmetric field are orthogonal to the $\boldsymbol{P}$ direction and are confined to meridional planes. as another axisymmetrical example, consider a poloidal field with field. lines covering the surfaces of a family of nested toruses. When a toroidal component is added to this field, field lines acquire an azimuthal slant so that instead of staying in planes of constant $\varphi$ they spiral around the axis or their torus, in a way resembling that found in toroidal plasma confinement devices.

Some of the elementary applications of (26) to dynamo theory have been described by Elsasser [1956]. For instance, in a conducting sphere both components of (26) will tend to decay with time: differential. 
rotation - such as is observed on the sun and Jupiter, both of which have magnetic fields - will amplify the toroidal component, but the strength of this process is proportional to the poloidal field which ultimately decays, unless it is continuously maintained by some leedback mechanism originating in the toroidal component. Pariker [1955] showed that radial Ilow in the rotating Irame may induce cyclonic swirling which indeed leads to such feedback. Various theories of solar magnetism based on such considerations have been advanced [Babcock, 1961; Leighton, 1969] but the details are beyond the scope of this review.

\section{(b) VECTOR POIENTIAL FOR A CURL-FREE FIEID}

T'wo identities userul in handling (26) are

$$
\begin{aligned}
\nabla \times \psi_{I} \underline{r} & \equiv \nabla \psi_{1} \times \underline{r} \\
\nabla \times \nabla \times \psi_{2} \underline{r} & \equiv \nabla \partial / \partial r\left(r \psi_{2}\right)-\underline{r} \nabla^{2} \Psi_{2}
\end{aligned}
$$

If $\underline{B}$ is curl-free and satisfies (2), with $\gamma$ expanded as in (II) and (20), then it can be represented as a poloidal rield with $\psi_{2}$ a harmonic function simply related to $\gamma$ [Sterm, 1964]. To see that, note that iI. $\Psi_{2}$ is a series of spherical harmonics, the same holds true for $\partial / \partial r\left(r \psi_{2}\right)$. Furthermore, the last term of (28) vanishes in that case, so that only the gradient of a harmonic function remains. This allows formal identirication

$$
\gamma=-\partial / \partial r\left(r \psi_{2}\right)
$$

and a term-by-term comparison of expansion coeificients. Specifically, if $\gamma$ has expansion coefricients $\left(g_{n}^{m}, h_{n}^{m}, \vec{g}_{n}^{m}, \vec{h}_{n}^{m}\right)$ as in (11) and (20) and $\Psi_{2}$ has corresponding coefficients $\left(G_{n}^{m}, H_{n}^{m}, \vec{G}_{n}^{m}, \overrightarrow{\mathbb{H}}_{n}^{m}\right)$, then 


$$
\begin{aligned}
& \mathrm{G}_{\mathrm{n}}^{\mathrm{m}}=\mathrm{g}_{\mathrm{n}}^{\mathrm{m} / \mathrm{n}} \quad \mathrm{H}_{\mathrm{n}}^{\mathrm{m}}=\mathrm{h}_{\mathrm{n}}^{\mathrm{m} / \mathrm{n}} \\
& \overrightarrow{\mathrm{G}}_{\mathrm{n}}^{\mathrm{m}}=-\overline{\mathrm{g}}_{\mathrm{m}}^{\mathrm{n}} /(\mathrm{n}+1) \quad \overline{\mathrm{H}}_{\mathrm{n}}^{\mathrm{m}}=-\overline{\mathrm{n}}_{\mathrm{n}}^{\mathrm{m}} /(\mathrm{n}+1)
\end{aligned}
$$

To obtain the vector potential A for a given $\gamma$. expanded as in (11) and (20), equations (30) are used to derive the expansion terms of $\psi_{2}$, alter which one calculates

$$
\underline{A}=\nabla \times \Psi_{2} \underline{r}
$$

\section{(c) MODEIS OF THE MAGNETOSPHERE}

Curl-Iree models of the Iar-away geomagnetic ilield, as were described in the section on the scalar potential, are incapable or great quantitative accuracy, because the region wich they describe contains an appreciable current density. As an alternative, Mead and Fairfield [1975; Fairielield and Mead, 1975] tried to represent the field in such regions by expanding each component of $\underline{B}$ in powers of cartesian coordinates

$$
B_{i}=\sum_{n, r, s} a_{i n r s} x^{r} y^{s} z^{n-r-s}
$$

Potential ílelds such as those of (20) are easily reduced to this Form but they comprise only a limited subclass of such Iields.

Two problems, arise, however: the İirst is how to assure the vanishing or $\nabla \cdot \underline{B}$, and the second is the dificiculty in controlling the model near the boundary of the region for which it is derived, due to the pre of positive powers of $x, y$ and $z$.

To overcome the first difiticulty Mead and Fairfield derived the relations between the coeficients of (32) which are required in 
order Ior $\boldsymbol{\nabla} \cdot \underline{B}$ to vanish. They then analyzed magnetometer data from space and derived a "best ritting set" of coefficients for (32) by a least-squares procedure similar to the one used for deriving the main field, described in section $(2-i)$, but based on vector data. In that calculation the relations which assure the vanishing of $\boldsymbol{\nabla} \cdot \underline{B}$ were introduced as constraints and were handled by the method of Lagrange's multipliers.

A simpler method is however available: noting that

$$
\underline{x}=\underline{\hat{x}} x+\hat{\underline{y}} y+\underline{\hat{z}} z
$$

and expanding (26) in cartesian coordinates shows that if $\psi_{1}$ and $\psi_{2}$ are expanded in the form (32), then the resulting components $B_{i}$ also have this form. In particular, if the largest power $n$ of. variables having the dimension of length is $N$ ior $\psi_{I}$ and $\mathrm{N}-1$ for $\psi_{2}$, then its value for the expansion of $B_{i}$ will be $N$.

Not all terms in the expansions of $\psi_{i}$ produce independent contributions to $\underline{B}$, because any part of $\psi_{i}$ which depends on $r$ alone does not aifect $\underline{B}$. The terms with $n=0$ belong to this class and should therefore be omitted. Consider next the three quadratic terms proportional to $x^{2}, y^{2}$ and $z^{2}$. These can be combined to give 3 independent terms proportional to $x^{2}, y^{2}$ and $r^{2}$ and the last of these does not contribute to $\underline{B}$, so that it can be omitted. Similar arguments show that for any integer $k$, one coefíicient can be eliminated among those representing homogeneous polynomials of degree, $2 \mathrm{k}$ in the expansion or $\psi_{1}$ or $\psi_{2}$. 
One advantage of this approach is that no precautions are required to ensure the vanishing of $\nabla \circ \underline{B}$. It is also easily generalized to overcome the second difíiculty mentioned earlier, by devising models in which $\psi_{1}$ and $\psi_{2}$ are expanded in the form

$$
\psi_{j}=\sum_{n, m, s} a_{j n m s} x^{m} y^{s} z^{n-m-s} \exp \left(-r / r_{0}\right)
$$

where $r_{0}$ is some chosen scale distance (in prineiple, several series with different choices of $r_{0}$ could be used). It is more convenient to use in this case spherical harmonics

$$
\psi_{j}=\sum_{n, m, s} r^{n} \exp \left(-r / r_{0}\right) p_{m}^{s}(\theta)\left[u_{j n m s} \cos s \varphi+v_{j n m s} \sin s \varphi\right]
$$


from which the components of $\underline{B}$ are readily derived. It should be noted that the expansion of (34) is not equivalent to that of (35); in fact, the latter expansion is equivalent to

$$
\psi_{j}=\sum_{n} r^{n} \sum_{u, v, w} a_{\text {jnuvw }}(x / r)^{u}(y / r)^{v}(z / r)^{w} \exp \left(-r / r_{0}\right)
$$

which includes the terms $0 \vec{I}^{\prime}$ (34) if $u+v+w=n$ but also contains additional expressions. Some comparisons between this method and that of Mead and Fairfield have been períormed (D. Stern, unpublished); while the improvement is rather small compared to the inherent dispersion of the observational data, this approach should be useful in future studies by providing more flexible analytical expansions.

Magnetospheric models somewhat similar to those described here have been used by olson and Pfitzer [1974]. However, the derivation of these models does not involve $\psi_{i}$ : it starts by fitting a system of currents to observed fields [ㅇson, 1974] and then approximates the cartesian components of their fields by general expansions similar to (32) and (34). The resulting models are not automatically divergenceIree.

It should be stressed that there exists no assurance that "global" models, representing the entire magnetosphere by one series of analytic functions of a given form, can do so with arbitrary accuracy. The power series (32), for instance, is an ordinary Taylor expansion and can represent functions only in a certain neighborhood of the origin, where it converges: this region may not only be inite, it may be smaller than the magnetosphere, in which case any representation of the rield in this way contains a finite error. The representation (34) appears at first sight to be more useful but it, too, is subject to the same restrictions since it just represents the cartesian expansion or $\psi_{i} \exp \left(r / r_{0}\right)$. 
At present, observational inaccuracies are of the same order as those introduced by global models and such models are therefore in general use. Ultimately, however, it may be necessary to adopt a difierent approach. Two extreme cases could then be considered - an extension of the global method by which the magnetosphere is divided into a number of large regions, each with its own expansion, and numerical representation by means of a tabulated network or values (e.g. of $\psi_{i}$ ). It should be realized, however, that the numerical representation can also be viewed as a local expansion, by means of the interpolation formulas used, and by making the numerical grid more sparse and the interpolation formula more powerful, the gap between these two extremes could in principle be bridged.

\section{(a) SPHERICAI VECTOR HARMONICS}

The spherical harmonic functions $P_{n}^{m}(\theta) \cos m \varphi$ and $P_{n}^{m}(\theta) \sin m \varphi$ - or, alternatively

$$
x_{n}^{m}=P_{n}^{m}(\theta) e^{i m \varphi}
$$

(in the notation of Morse and Feshbach [1953], p. 1898-1900, which this section will adopt) - form a complete set in which arbitrary analytic functions of $(\theta, \varphi)$ can be expanded. Similarly, spherical vector harmonics are 3 sets of vector functions of $\theta$ and $\varphi$, denoted as $\underline{P}_{n, m}, \underline{B}_{n, m}$ and $\underline{C}_{n, m}$, which find use in the expansion of vector functions.

Many problems in wave propagation lead to a vector rield $\underline{V}$ represented as in (23) and satisfying (24) $\vec{i} \omega$ s some values of $k$. This gives 3 independent equations of the form (25) and separation of the $r$ variable shows then that $\psi_{i}$ have the form

$$
\psi_{i}=\sum_{n, m} A_{n m i} F_{n m}(r) x_{n}^{m}(e, \psi)
$$


where $F_{n m}(r)$ stands for Hankel functions if is $\neq 0$ and powers of $r$ if $\mathrm{k}=0$. When (37) is then substituted in (23) it is found that all terms are expressed as the product of functions of $r$ with some member of one of the following three families of spherical vector harmonics

$$
\begin{aligned}
& \underline{P}_{n, m}=\underline{r} x_{n}^{m}(\theta, \varphi) \\
& \underline{B}_{n, m}=r[n(n+1)]^{-1 / 2} \nabla x_{n}^{m}(\theta, \varphi) \\
& \underline{C}_{n, m}=[n(n+1)]^{-1 / 2} \nabla \times \underline{r}_{n}^{m}(\theta, \varphi)
\end{aligned}
$$

Note that any dimensional dependence on $r$ in the above derinitions cancels out and also, by (27), that for any pair of values of the indices $\mathrm{n}$ and $\mathrm{m}$ the three above vectors are orthogonal. Other features of these vectors (and of their real and imaginary parts, since by (36) they represent complex quantities) are described by Morse and Feshbach [1923].

Many of the representation discussed earlier can be easily expressed in terms of spherical vector harmonics. For instance, the expansion (35) is formally the same as that of (37) and therefore the results of its substitution in (26) can be expressed in terms of the vectors listed in (38). 


\section{(4) EUTER POTENITALS}

\section{(a) DEFINITION AID GERERAL PROPERTIES}

An intuitive description of a magnetic iield, Iirst introduced by Michael Faraday, is provided by magnetic field lines (or "lines or îrce" as he termed them) - Iines which are everywhere tangential to $\underline{B}$. Such a description is completely analogous to the representation of the velocity field ol an incompressible fluid by means of streamlines.

For a long time fiela lines were mainly used as a device for visualizing the magnetic ileld. However, with increasing interest in particle motion and transport properties in a near-collisionless plasma - both in space and in the laboratory - Iield 1ines assumed new importance, since both these erfects are channeled along them. This created interest in mathematical descriptions of the magnetic lield which explicitely contain representation by field lines; such a description has been available in fluid dynamics and it involves two scalar functions $\alpha$ and $\beta$ such that

$$
\underline{B}=\nabla \alpha \times \nabla \beta
$$

The runctions $(\alpha, \beta)$ are generally known as Euler potentials because it was Leonhard Euler who first introduced such a representation in the context of Iluid dynamics; they appear to have been introduced into plasma physics by Grad [Northrop and Teller, 1960; Gardner, 1959]. A short review of their history and properties was given by Stern [1970] and the reader is referred to that work for details extending beyond the present brief description.

The basic properties of $(\alpha, \beta)$ are easily derived from (39). We have

$$
\begin{aligned}
& \underline{B} \cdot \nabla \alpha=0 \\
& \underline{B} \cdot \nabla \beta=0
\end{aligned}
$$


This means that surfaces of constant $\alpha$ and $\beta$ are everywhere tangential to $\underline{B}$ - and this property extends to lines along which two of such surfaces intersect, which thus are rield lines. Note that (39) implies (40), but not vice versa. Two Iunctions ( $u, v$ ) with the property or $(40)$

$$
\underline{B} \cdot \nabla u=\underline{B} \cdot \nabla v=0
$$

do not satisiy (39), in the general case, but rather

$$
\underline{B}=w(\nabla u \times \nabla v)
$$

where $w$ is an arbitrary runction of $(u, v)$. Functions such as $(u, v)$ may be called unmatched Eulcr potentials and have been introduced by Sweet [1950] and Dungey [1958]. They are conserved along rield Iines and are thereiore Iunctions of $(\alpha, \beta)$.

In general (subject to restrictions of uniqueness and single valuedness noted later) rield lines of a given configuration form a two-parameter ramily, in which the rield line corresponding to the values $\left(\alpha, \beta_{0}\right)$ of the parameters are represented by the intersection of the suriaces

$$
\begin{aligned}
& \alpha(x, y, z)=\alpha_{0} \\
& \beta(x, y, z)=\beta_{0}
\end{aligned}
$$

As an example, a simple nodel of the geonagnetic rield is given by an axial dipole of moment $B_{1}^{0}$; if (as in eq. 1l) a represents the earth's radius, one convenient choice of $(\alpha, \beta)$, in spherical coordinates $(r, \theta, \varphi)$, is given by

$$
\begin{array}{ll}
\alpha=a g_{1}^{0}(a / r) \sin ^{2} \theta & (42-a) \\
\beta=a \varphi & (42-b) .
\end{array}
$$


Given any two numerical values $\left(\alpha_{0}, \beta_{0}\right)$ of $\alpha$ and $\beta$, a fiela line of this field is derined by (4I). Note that (42-b) indicates that field lines lie in constant meridional planes, while $(42-a)$ gives the shape of a Iield line within such a plane. Other examples will be presented later.

Eulex potentials are not uniquely defined: for instance, $\alpha$ can be incremented by any function of $\beta$ without (39) being violeted. More generally, $(\alpha, \beta)$ may be replaced by $\left(\alpha^{\prime}, \beta^{\prime}\right)$, provided the new Euler potentials are iunctions of the old ones and the Jacobian of the transformation is unity:

$$
\partial\left(\alpha^{\prime}, \beta^{\prime}\right) / \partial(\alpha, \beta)=1
$$

Given a magnetic Iiela represented as in (40), it, is generally possible to choose for $\alpha^{\prime}$ (at least inside a restricted region in space) any well-behaved Iunction $\alpha(\alpha, \beta)$ and then derive a corresponding conjugate Euler potential $\beta^{\prime}$. An application of this property will be presented at the end of this section.

A vector potential corresponding to (39) is

$$
\underline{A}=\alpha \nabla \beta
$$

and it has the property $\underline{A} \cdot \underline{B}=0$ 。

With all these useful properties, there exist two important drawbacks to Euler potentials which limit their application. The first is non-linearity: the representation (39) is non-linear, since it involves products of the derivatives of $\alpha$ and $\beta$. This means that superposition does not hold For Euler potentials: given the Euler potentials for two fields $\underline{B}_{1}$ and $\underline{B}_{2}$, the functions $(\alpha, \beta)$ for the combined field does not equal the sum of the Euler potentials expressing $\underline{B}_{1}$ and $\underline{B}_{2}$ separately and, indeed, might be difficult to derive, even when the Euler potentials of the component fields are known. In practice this greatly limits the class or fields for which analytic iorms or even analytic approximations of $(\alpha, \beta)$ are readily derived. 
The other drawback, more important in the laboratory than in space applications, is the possibility that the labeling of rield lines by $(\alpha, \beta)$ is not single valued. In toroidal confinement devices, for instance (tokamaks, stellarators), î a íleld line is labeled by ( $\alpha, \beta$ ) in some limited region of the Iield and is then followed outside this region, it may litimately enter the region once more. In iact, such a ijield line usually returns to the same region again and again and with each return it coincides with some previously labeled field line - in general, one with difierent values of $(\alpha, \beta)$. In such cases the labeling is single valued only if a limited region is considered and is not possible when the entire Iield is represented. For Iurther details about these and other properties of Euler potentials the reader is referred to the review by stern $[1970]$.

As an illustration of an application consider the motion of trapped particles in the magnetosphere under the combined influence of a static electric field

$$
\underline{E} \quad=-\nabla \phi
$$

and the geomagnetic field $\underline{B}$. Because of the high conductivity along iield lines the electric rield (except in some special regions, perhaps) comes close to being orthogonal to $\underline{B}$, i.e.

$$
\underline{B} \cdot \nabla \phi=0
$$

By (40) this condition is neatly expressed by the requirement that $\phi$ depends only on $(\alpha, \beta)$

$$
\phi=\phi(\alpha, \beta)
$$

A charged particle of very low energy, conserving the two lowest adiabatic invariants but with negligible magnetic drift, will move in this field with the drift velocity

$$
\underline{v}_{t}=(\underline{B} \times \nabla \phi) / B^{2}
$$


From this $\underline{\mathrm{V}}_{\mathrm{E}} \cdot \nabla \phi=0$ and therefore the particle stays on a single equipotential suriace throughout its motion.

Now as stated earlier, any "well-behaved" function $\alpha$ of $(\alpha, \beta)$ can be chosen to replace $\alpha$ and an appropriate "conjugate potential" $\beta$ " can then be Iound Ior it. In particular, let the role of $\alpha$ ' be played by $\phi(\alpha, \beta)$ and let the conjugate potential be denoted $\psi(\alpha, \beta)$, i。e.

$$
\underline{B}=\nabla \phi \times \nabla \psi
$$

It is instructive to derive the rate at which $\psi$ changes at the location of the drifting particle. By (47) and (48)

$$
\mathrm{a} \psi / \mathrm{dt}=\underline{\mathrm{v}}_{\mathrm{E}} \cdot \nabla \psi=1
$$

i.e.

$$
\psi=t-t_{0}
$$

Thus a swarm of particles starting from a surface or constant $\Psi$ at $t=0$ will always share the same value or $\psi$. The averaged motion of such particles - that is, the gradual change of their guiding field lines is conveniently studied in the $(\alpha, \beta)$ plane, where to every pair of values of the Euler potentials - and hence, to every possible guiding ilield line - there corresponds one point (in the geomagnetic iield the equatorial plane can play such a role).In this plane the lines

$$
\phi(\alpha, \beta)=\text { constant }
$$

can be viewed as rays along which very low energy particles propagate, while lines or constant $\psi$ act as "waverronts" marking the particles" progress. Figure (1), taken from Stern [1974] gives lines of constant $\phi$ (solia) and $\psi$ (broken) for some particular geoinagnetic model, in which the earth's $\vec{i} i e l d$ is approximated by a dipole field and some analytical model of $\phi$ is assumed. 
The preceding is readily extended to particles of finite energy conserving the magnetic moment $M$ and the second invariant $J$, except that now $\phi$ is replaced by the averaged guiding center hamiltonian $\mathrm{K}(\alpha, \beta, \mathrm{M}, J)$ introducea by Northrop and Teller 1960 This generalization has been developed for the dipole ileld by Chen and Stern $[1972]$, who provided an analytical approximation for $\mathrm{K}$ and expressed its conjugate potential by means of numerical integration.

\section{(b) EXAMPLES}

In general, simple analytical Iorms for the Euler potentials can only be iound for classes or ilelds with some type or symmetry. For instance, two-dimensional fields or the form

$$
\underline{B}=B_{x}(x, y) \underline{\hat{x}}+B_{y}(x, y) \hat{y}
$$

have Euler potentials

$$
\begin{aligned}
& \alpha=\int B_{x} d y+I(x)=-\int B_{y} d x+g(y) \\
& \beta=z
\end{aligned}
$$

where the runctions $\hat{I}(x)$ and $g(y)$ are obtained by deriving $\alpha$ in two difierent ways (because $\boldsymbol{\nabla} \cdot \underline{B}=0$, two such equivalent derivations are possible). The hamiltonian for a particle with mass $m$ and charge $q$ moving in such a Iield can then be written

$$
H=(1 / 2 m)\left[p_{x}^{2}+p_{y}^{2}+\left(p_{z}-q \alpha(x, y)\right)^{2}\right]
$$

Since $p_{z}$ is a constant of the motion it follows that the motion reduces to that of a two-dimensional íree particle in a non-negative potential

$$
v=\left(p_{z}-q \alpha\right)^{2} / 2 m
$$


For all such motions, lines of constant $\alpha$ in the $(x, y)$ plane which trace the pattern of Iiela lines - are equipotentials of $V$. The value of $V$ attached to any such line and the general configuration of regions of high and low potential depends on the constant $\mathrm{p}_{\mathrm{z}}$ appropriate for the given motion. One interesting conigiguration of this class occurs in the neighborhood of an X-type neutral line [Russbridge, 1971]; other examples and generalizations have been examined by stern [1975].

Axisymmetrical poloidal fields, by (26), can be represented by Euler potentials with $\beta=\varphi$, since

$$
\begin{aligned}
\nabla \times \nabla \times \underline{r} \psi_{2}(r, \theta) & =-\nabla \times \underline{\hat{\varphi}}\left(\partial \psi_{2} / \partial \theta\right) \\
& =-\nabla\left[r \sin \theta\left(\partial \psi_{2} / \partial \theta\right)\right] \times \nabla \varphi
\end{aligned}
$$

The aipole example oi eqs. (42) belongs to this class. It is also possible to derive simple Euler potentials for pure toroidal rields:

$$
\nabla \times \Psi_{2} \underline{\underline{r}}=\nabla \Psi_{1} \times \nabla r
$$

However (as noted earliex) when fields of both kinds are combined no simple way oi deriving Euler potentials exists.

A case of practical interest involves the main geomagnetic ifield, usually represented by a scalar potential. $\gamma$ as in eq. (1.1). No analytical method is known by which $(\alpha, \beta)$ may be simply derived for such a field, but a perturbation technique is available for obtaining them approximately.

The method is based on the observed property that the dipole component $g_{I}^{0}$ in (11) dominates the expansion, especially if $\gamma$ is expressed in dipole cuordinates. In such coordinates let $\left(\alpha_{0}, \beta_{0}\right)$ be the dipole Euler potentials of eqs. (42) (the subscripts zero reier here to zero-order approximations and are unrelated to those appearing 
in equations 41 ), while $\gamma_{0}$ is the corresponding scalar potential. Iet $\left(\alpha_{1}, \beta_{1}, \gamma_{1}\right)$ be the Iirst-order corrections, linear in the higher harmonic coefijcients $\left(\mathrm{g}_{n}^{\mathrm{m}}, \mathrm{h}_{n}^{\mathrm{m}}\right)$, applied to these three quantities. Substitution in (39) then gives

$$
-\nabla \gamma_{1}=\nabla \alpha_{0} \times \nabla \beta_{1}+\nabla \alpha_{1} \times \nabla \beta_{0}
$$

Irom which

$$
\begin{aligned}
& \nabla \alpha_{2} \cdot \nabla \gamma_{0}=\nabla \alpha_{0} \cdot \nabla \gamma_{1} \\
& \nabla \beta_{1} \cdot \nabla \gamma_{0}=\nabla \beta \gamma_{0} \cdot \nabla \gamma_{1}
\end{aligned}
$$

Expressing $\left(\alpha_{1}, \beta_{1}, \gamma_{1}\right)$ in terms of $\left(\alpha_{0}, \beta, \theta\right)$ allows these equations to be integrated and provides $\left(\alpha, \beta_{1}\right)$ within arbitrary Iunctions of $\left(\alpha_{0}, \beta_{0}\right)$. However, (57) contains only two of the three scalar relations implied by the vector equation (56); if the remaining scalar relation is now invoked, the arbitrary added iunction may be determined. Details have been derived by Stern $[1967]$ and related expressions were iirst obtained in a difierent context by Pennington $[1961,1967]$ - The calculation has not been extended to higher order, except for one eifort by Hassit [1967] in which second-order unmatched Euler potentials were derived.

The same perturbation method can also be applied to the external harmonics of section (2-8) and in particular to the simple model described there, with the expansion or $\gamma$ involving only the three coeficients $g_{1}^{0}, \vec{g}_{1}^{0}$ and $\vec{g}_{1}^{2}$. As might be expected, the resulting expansion breaks down at large distances, but it also becomes unrealistic near the $z$ axis. It provides a Iairly good representation of the field in the regions occupied by trapped particles; in the noonmidnight cross section of the $(\alpha, \beta)$ modefin the region in which the model field departs markedly Irom the curlarree ileld from which it was derived, there exists the added reature (not found in the curl-free model) of a neutral point on the night side of the dipole, in addition to two such points on the day side, corresponding to the polar cusps. Further details have been given by stern $[1967]$ and Kosik $[1971 \mathrm{a}, \underline{\mathrm{b}}]$. 
A model with some similar properties (but much smaller deformation) has been developed by Parker [1960].

While accurate representations of the magnetospheric field by means of $(\alpha, \beta)$ are difficult to obtain and no iterative procedures for deriving them have been published, it is relatively easy to devise simple models which exhibit appropriate qualitative properties. For instance, a distor ted dipole field, compressed on one side and stretched out on the other, is given by

$$
\begin{aligned}
\alpha / a^{2} g_{1}^{0} & =\sin ^{2} \theta / x-k \sin ^{6} \theta(1-\cos \varphi)^{3} \\
\beta & =a \varphi
\end{aligned}
$$

where $k$ is an adjustable constant. It is easy to distort this model further, in such a way that all its field lines are contained within the surface

$$
f(r, \theta, \varphi)=1
$$

This is accomplished if $\alpha$ is multiplied by $(1-f)$; in order that the field will continue to approximate the dipole near the origin, $I$ should tend to zero as $r \rightarrow 0$. For instance, if the field is to be contained inside the paraboloid

$$
r=r_{0} /(1+\sin \theta \cos \varphi)
$$

one can multiply $\alpha$ of (58) by the iactor

$$
1-\left(r / r_{0}\right)^{m}(1+\sin \theta \cos \varphi)^{m}
$$

Increasing the adjustable parameter $m$ narrows down the transition zone between the main field and the boundary field. Further improvement can be obtained by shilting the focus of the paraboloid away from the origin, but it should be noted that in 211 these models the boundâry is always connected to the two dipole rield lines which emerge along the $z$ axis.

As a rinal note, axisymmetric models oI this sort have been constructed by Barish et al. [1974] for the magnetic field of Jupiter, lollowing its observation by Pioneer 10 [S Smith et $21 ., 1914]$. 


\section{(c) THE I PARAMETER}

Before the $(\alpha, \beta)$ system for the magnetosphere was developed an alternative approach to the labeling of nagnetic ilield lines was introduced in the form of the so-called I parameter due to McIlwain $[1961,1966]$.

As an intuitively meaningiul quantity the I parameter is extremely userul: it has dimensions or length in units or earth radii and in a dipole field its value at any given point equals the maximum distance from the dipole attained by the field line passing the given point. In a perturbed dipole rield (and the parameter is only delined ror dipole fields and perturbed dipole rields) the value $\tau(\underline{r})$ at some given position $\underline{r}$ still approximately equals the largest distance irom the origin which is attained by the Iield line through $\underline{x}$. However, the generalization of $L$ to perturbed dipole Iields is not periormed in a way which aims at preserving the equatorial crossing distance. Instead, the definition tries to preserve a different property, useful in studies of trapped radiation - that î a charged particle is trapped along any rield line with a given value of $I$, as it gradually drifts around the dipole then all field lines which it passes will have the same value of I.

This property is only approximately achieved. Indeed, it cannot in general hold for all particle orbits in an asymetric rield, since two particles starting on a given Iield line in such a field with dirierent mirroring points will in general follow slightly difrerent surfaces ("drift shells". during their drift (this is known as shell splitting). However, for the geomagnetic field the separation between such surfaces tends to be small and consequently the values oI I encountered by a particle in its drift generally vary by less than $2 \%$.

In this section the relation between $L$ and $(\alpha, \beta)$ will be traced, the inherent limitations or $I$ will be clariried and suggestions will be described ior ways in which the concept can be generalized. 
In a static near-dipole magnetic íield, trapped particles tend to preserve the two lowest adiabatic invariants (the third invariant is not needed here)

$$
\mu=p_{\perp}^{2} / 2 n B \quad J=\oint p_{h} a l
$$

where $m$ is the rest mass, $\left(p_{1}, p_{1}\right)$ are the components of the momentum parallel and perpendicular to $\underline{B}$ and integration is along a field line. Since the magnitude $p$ of the momentum is also conserved (in the absence or electric rields, which will now be assumed) it is possible in this case to replace $(\mu, J)$ by two related quantities $\left(B_{\mathrm{In}}\right.$, I) which are also constants of the motion but do not depend on the particle's energy:

$$
\begin{aligned}
& B_{m}=p^{2} / 2 m \mu \\
& I=J / 2 p=\int_{B_{m}^{\prime}}^{B_{m}}\left(1-B / B_{m}\right)^{1 / 2} a l .
\end{aligned}
$$

where the integration of I extends between mirror points at which $\mathrm{B}=\mathrm{B}_{\mathrm{m}}$. As the particle drifts from one guiding field line to the next, it always chooses that one of the adjacent field lines on which the value of $I$, evaluated between fixed values of $B_{m}$, is the same.

As the particle drifts its guiding rield lines gradually trace a surface - a closed suriace for trapped particles - called a drift shell or a drift suriace. Because drift shells are tangential to $B$ their equations have the form

$$
I(\alpha, \beta)=\text { constant }
$$

For each pair of parameters $\left(I, B_{m}\right)$ there exists a drift shell and thereiore the entire collection of drift shells can be represented by some Iunction $F$ as 


$$
F\left(\alpha, \beta, I, B_{m}\right)=\text { constant }
$$

(the constant may be chosen as 0 or $I$ without loss or generality). Isolating $\alpha$ gives

$$
\alpha=G\left(I, B_{m}, \beta\right)
$$

In a dipole lield $\beta$ may be eliminated because of axial symmetry; in that case, if $\left(\alpha_{0}, \beta_{0}\right)$ are the Euler potentials derined in (42), then (65) reduces to

$$
\alpha_{0}=G_{0}\left(I, B_{m}\right)
$$

Now $\left(I, B_{m}\right)$ can be replaced as parameters characterizing drirt shells by any two well-behaved Iunctions depending on them [stern, 1968]. In particular, in the case of the perturbed dipole field, $G_{0}\left(I, B_{m}\right)$ or any function $I\left(G_{0}\right)$ can be used as one such parameter, while $B_{m}$ may be retained as the second one. This characterization will have the advantage that in the dipole limit - due to (66) - the shell equation depends only on a single parameter $\mathrm{G}_{0}$ or. $\mathrm{L}\left(\mathrm{G}_{0}\right)$, not on two. In that limit all the trajectories starting from the same initial field line trace the same surface and have identical values or $G_{0}$ or $I\left(G_{0}\right)$; by way of contrast, i.I such shells were classified by using ( $I, B_{m}$ ), a finite range in both these parameters would be required.

In a perturbed dipole field the shell equation is given by (66) and no single-parameter description is possible. However, ir the perturbation is small, equation (65) will differ from (66) only by small correction terms. Denoting such terms by subscript " 1 " and using $\left(G_{0}, B_{m}\right)$ as parameters brings (65) to the form

$$
\alpha=G_{0}+G_{1}\left(G_{0}, B_{m}, \beta\right)
$$


Thus the shell's equation depends strongly on $G_{0}$ and only weakly, through the correction term $G_{I}$, on the second parameter $B_{m}$. If $G_{I}$ is ignored - or better, replaced by $\left\langle G_{3}\right\rangle$, its average over $B_{m}$ then all particles characterized by some value of $G_{0}$ will stay close to the suriace

$$
\alpha=G_{0}+\left\langle G_{1}\left(G_{0}, \beta\right)\right\rangle
$$

and thus their drift shell will still be approximately characterized by the single parameter $G_{0}$. All the preceaing also holas it $G_{0}$ is replaced by $I\left(G_{0}\right)$; in particular, McIlwain's desinition of $L$ [McIIwain, $1961]$ is equivalent to

$$
L\left(I, B_{m}\right)=a g_{I}^{0} / G_{0}\left(I, B_{m}\right)
$$

In McIlwain's work the above Iunction is approximated in two steps, beginning with the derinition of uxiliary Iunctions

$$
\begin{aligned}
& \mathrm{Y}=\ln \left(\mathrm{I}^{3} \mathrm{~B}_{\mathrm{m}} / \mathrm{g}_{1}^{0}-1\right) \\
& \mathrm{X}=\operatorname{In} \xi \text { where } \xi=I^{3} \mathrm{~B}_{\mathrm{m}} / \mathrm{g}_{I}^{0}
\end{aligned}
$$

The relation between $\mathrm{X}$ and $\mathrm{Y}$ is then approximated by a polynomial

$$
Y=\sum_{n=0}^{\mathbb{N}} a_{n} x^{n}
$$

In the original derivation [McIlwain, 1961] sixth-order polynomials were used and different expansions were chosen for each of 5 ranges of X. Later on [McIIwain, 1966] an improved approximation was derived, with $\mathbb{N}$ increased to 9 and the number of ranges to 6 , and an inverse expansion, expressing $X$ in terms or $Y$, was also provided. A much simpler and fairly accurate approximation has been proposed by Hilton [1971] , who matched the analytical behavior of $I$ at the limits $I=0$ and $\mathrm{B}_{\mathrm{m}} \rightarrow \infty$ with that of the approximation. His result is 


$$
L^{3} B_{m} / B_{1}^{0}=1+a_{1} \xi^{1 / 3}+a_{2} \xi^{2 / 3}+a_{3} \xi
$$

where

$$
\begin{aligned}
a_{1} & =3(2)^{1 / 2} / \pi \approx 1.3507 \\
a_{2} & =\left[2+(3)^{1 / 2} \ln \left(2+3^{1 / 2}\right)\right]^{-3} \\
& \approx 0.0475455 \\
a_{3} & =0.456376
\end{aligned}
$$

The relative inaccuracy $\Delta I / L$ or this approximation is $10^{-4}$ or less.

The I parameter is widely used in labeling lield lines, in the Following manner. Given a point $P=(x, y, z)$ in the earth's ileld (which is assumed to be given by the expansion (11) of the scalar potential), the integral $I$ is derived ior particles mirroring at $P$, by numerical integration. Using one of the Iormulas described earlier, $I\left(I, B_{m}\right)$ is derived for these particles and its value is regarded as a labeling parameter for the field line passing $\mathrm{P}$. Of course, what has been derived here is an approximation to $a g_{1}^{0} / \alpha(x, y, z)$ where $\alpha$ is the pexturbed-dipole generalization of $\alpha_{0}$ in $(42-a)$ (in principle a $g_{1}^{0} / \alpha$ could be chosen as a new Euler potential $\alpha^{\prime}$, but the Iorm or $\beta$ ' is then complicated). The derivation of this approximation is encumbered by the need for a numerical integration expressing $I$, although this integration has been simplified - as far as computer use is concerned - by G. Kluge (ESRO Internal Note 66, October 1970) who Iirst transformed it by inversion with respect to the origin.

This correspondence between $I$ and $\alpha$ works both ways and stern [1968] has used approximations of $\alpha$ for perturbed dipole fields to obtain approximations to $I\left(I, B_{m}\right)$, avoiding the need Ior deriving I by line integration. This method is already implicit in the work of Pennington $[1961,1967]$ who derived the equations of dritt shells in a perturbed dipole iield by a straightiorward perturbation method. 
poloidal

The I parameter can be generalized to any near-axisymmetric $\Lambda^{\text {magnetic }}$ Iield : if (65) represents the Iamily of drift shells in such a field, it is only necessary to derive the corresponding equation (66) Ior the underlying axisymmetric Iield and then choose $G_{0}$ or some Iunction of $G_{0}$ as the $I$ parameter.

Such an approach was applied by Stem $[1968]$ to the 3-parameter model of the magnetosphere discussed in the preceding section. At moderate dis tances Irom the origin this Iield may be viewed as a perturbation of the axisymmetrical iield given by

$$
\begin{gathered}
\underline{B}=-\nabla \gamma \\
\gamma=a\left[g_{I}^{O}(a / x)+\bar{g}_{I}^{O}(r / a)^{2}\right] \cos \theta
\end{gathered}
$$

By a perturbation method Stern derived an approximate form for the equation corresponding to (66) in this field

$$
\alpha=G_{0}^{\prime}\left(I, B_{m}\right)
$$

and denoted $I^{\prime}=a g_{I}^{O} / G^{\prime}$ as the generalized I-parameter. In principle the same generalization should be feasible for taking into account the axisymmetrical ring current of the earth, for treatment of particle motion in slightly asymmetrical mirror machines and for similax applications.

A number of quantities related to $I$ are in general use. Among the best known of these is the invariant latitude $\Lambda$ : given a point with some value of I, its "invariant latitude" satisfies

$$
\cos ^{2} \Lambda=1 / \mathrm{L}
$$

The underlying idea is that $i \hat{I}$ all points in the perturbed ileld were to be mapped to a dipole field in a way that $I$ (or $\alpha$ ) were conserved, then $\Lambda$ would be the latitude at which the field line through the point met the earth's surface. A similar generalization for the longitude is provided by the magnetic longitude [McIlwain, 1966]. 


\section{(5) LOCAL REPRESENTATIONS}

It is sometimes required to represent $\underline{B}$ in the immediate neighborhood of some given point $\mathrm{P}$ without regard to the Iield's conjiguration in the rest oI space. Two important cases in which this happens are in the guiding center motion of charged particles and in plasma dynamics near neutral points: it is useful in such cases to introduce so-called local representations of $\underline{B}$ which are valid only in a restricted neighborhood of $\mathrm{P}$.

The most common local representation - and the only one discussed here - is the Taylor expansion

$$
\underline{B}(\underline{r})=\underline{B}_{0}+\underline{r} \cdot \nabla \underline{B}_{0}+\frac{1}{2} \underline{r}: \nabla \nabla \underline{B}_{0}+\ldots
$$

where all quantities with subscript zero are evaluated at the point $P$, regarded as the origin $\vec{I}$ or $\underline{r}$. Such a representation is fully equivalent to (32) and is best handled by the methods developed in section (3-c), i.e. by expressing $\underline{B}$ in the form

$$
\underline{B}=\nabla \times \underline{r} \psi_{1}+\nabla \times \nabla \times \underline{r} \psi_{2}
$$

and expanding $\psi_{1}$ and $\psi_{2}$ in polynomials in $(x, y, z)$. Let $\psi_{i}^{(j)}$ denote the sum of those terms in the expansion of $\psi_{i}$ which form homogeneous polynomials of degree $j$ in $(x, y, z)$. Then it is easily seen that

$$
\begin{aligned}
\underline{B}_{0} & =\nabla \times \nabla \times \underline{r} \psi_{2}^{(1)} \\
\underline{r} \cdot \nabla \underline{B}_{0} & = \\
\frac{1}{2} r r: \nabla \nabla \underline{B}_{0} & =\nabla \times \nabla \times \underline{r} \psi_{2}^{(2)}+\nabla \times \underline{r} \psi_{1}^{(1)} \\
& \nabla \times \nabla \times \underline{r} \psi_{3}^{(3)}+\nabla \times \underline{r} \psi_{1}^{(2)}
\end{aligned}
$$

and so Porth, with the degree of the terms on both sides increasing 
by unity with each succeeding line. The coefficients on either side are not in general independent. On the leit side the terms are related by virtue of the condition $\nabla \cdot \underline{B}=0$; for instance, $\nabla \underline{B}_{0}$ is required to have zero trace, so that only 8 of its coepricients can be independently specified. On the right any combination of terms which depends on $r$ alone does not contribute to the rield and should be eliminated by the methods of section (3-c). The vector potential follows at once irrom (77) ; it should be noted that no simple relation exists between the expansion (75) of $\underline{B}$ and a similar one for $\underline{A}$.

In principle [Morse and Feshbach, 1953] an alternative local. expansion is possible in which $x$ in (76) is replaced by the constant unit vector $\underline{\hat{z}}$ :

$$
\underline{B}=\nabla \times \Phi_{1} \underline{\underline{z}}+\nabla \times \nabla \times \Phi_{2} \underline{\underline{z}}
$$

This, however, introduces a considerable ambiguity. In (76) no component of $\underline{B}$ can be both poloidal and toroidal, so that the assignment of such components to $\Psi_{1}$ and $\psi_{2}$ is never arbitrary. The analogous statement does not hold for $(78)^{2}$ and because of this, except perhaps for special cases, this expansion is not recommended.

Local Euler potentials are similarly expressed as polynomials of ascerding orders

$$
\begin{aligned}
& \alpha=\alpha^{(1)}+\alpha^{(2)} \ldots \\
& \beta=\beta^{(2)}+\cdots
\end{aligned}
$$

with

$$
\begin{aligned}
\underline{B}_{0} & =\nabla \alpha^{(1)} \times \nabla \beta^{(1)} \\
\underline{r} \cdot \nabla \underline{B}_{0} & =\nabla \alpha^{(1)} \times \nabla \beta^{(2)}+\nabla \alpha^{(2)} \times \nabla \beta^{(1)}
\end{aligned}
$$

and so forth. The calculation here is greatly simplified if the coordinate 
axes are suitably chosen, with the $z$ axis along $\underline{B}_{0}$ and

$$
\alpha^{(1)}=x \quad \beta^{(I)}=B_{0} y
$$

If then

$$
\begin{aligned}
& \alpha^{(2)}=a_{11} x^{2}+a_{22} y^{2}+a_{33^{2}} z^{2}+\left(a_{12}-2 b_{22}\right) x y+a_{13} x z+a_{23} y z \\
& \beta^{(2)}=b_{11} x^{2}+b_{22} y^{2}+b_{33} z^{2}+\left(b_{12}-2 a_{11}\right) x y+b_{13} x z+b_{23} y^{y z}
\end{aligned}
$$

it is round that the 4 coefricients $a_{11}, a_{22}, b_{11}$ and $b_{22}$ affect only orders higher than the rirst in (77), while the remaining 8 coeificients are uniquely related to the 8 independent terms which determine $\quad \nabla \underline{B}_{0}$.

An interesting local representation, equivalent to (Th) up to the order of $\nabla \underline{B}_{0}$, has been described by Siambis and Norihrop $[1966]$. At a point $P$ in the field let the unit vectors $(\hat{\underline{I}}, \hat{\underline{M}}, \hat{\mathbb{N}})$ form a right-handed orthogonal system with the arigin at $P, \hat{I}$ parallel to $\underline{B}$ and $\hat{M}$ pointing towards the center of curvature of the field line through $P$. In this system the components of $\nabla \underline{B}_{0}$ can be expressed in terms of curvature and shear coefficients (1oc. cit,, appendix) While the coeficients themselves can be expressed in terms of the spatial variation of the basic unit vectors following a shift in $P$. Some applications or such a system of local vectors exist in plasma theory, e.go in the work of Wilson $[1970]$.

The Taylor expansion (75) forms a focal point of the theory of neutral points $[$ Dungey, 1953, 1963]. A first-order neutral point exists at $P$ OI $\underline{B}_{0}$ vanishes but $\nabla \underline{B}_{0}$ does not, so that to lowest order, in the vicinity of $P$ 


$$
\underline{B}=\underline{r}_{0} \cdot \nabla \underline{B}_{0}
$$

Even though $B$ vanishes at $P$ there may exist singular field lines which pass through $P$ and at sufficiently small distances from $P$ such Iield lines can then be approxinated by straight lines. In the region of interest the magnetic field $\underline{B}$ depends linearly on $(x, y, z)$ as measured Irom $P$ and therefore, $i \vec{I} \underline{\xi}$ is the radial distance measured from $P$ along one of the singular lines, its value on that line should satisfy (to lowest order)

$$
\underline{B}=\lambda \underline{\xi}
$$

where $\lambda$ is some constant. By (82)

$$
\lambda \underline{\xi}=\underline{\xi} \cdot \nabla \underline{B}_{0}
$$

and thereiore $\lambda$ must be a real eigenvalue of $\boldsymbol{\nabla}_{\mathrm{B}_{0}}$. There may exist either 1 or 3 such eigenvalues; accordingly, there will exist 1 or 3 such singular ijeld lines and $P$ will be classiried as an o-type or X-type neutral point, so named because rield line configurations near $P$ resemble either the letter 0 or the letter $X$ (Figure 2).

A great amount of theoretical work and of interest has locused on X-type neutral points, since they play a central role in processes which change the topology or magnetic field lines; for a review the reader is referred to the work of Vasyliunas [1975]. Surprisingly, only limited experimental work exists on the subject [e.g. Baum et al., 1973], nor has there been much interest in magnetic field configurations in space with 0 -type neutral points. 


\section{AFTERWORD}

This is a draft of a review article and additional relevant material may be added to the rinal version. The author welcomes any suggestions for such additions as well as comments of a general nature.

Two additions which will be incorporated concern the description of two extraterrestrial magnetic fields by harmonic expansions similar to eq. (11). Altschuler and Newikirk [1969] applied such an expansion to the magnetic ifield of the sun and their work was extended by Schatten

[1971; Schatten and Howe11, 1971]: In addition, Acuna and Ness [1975] extracted a model of the extemal field of Jupiter from the observations of Pioneer 1.1, which passed close enough to the planet to allow the larger non-dipole harmonic terms to be estimated.

Another such addition concerns the work of Voigt $[1972]$, who developed a general class of current-iree magnetospheric models. In Voigt's approach the bounding suriace (i.e. magnetopause) is deduced Irom observations and serves as a boundary condition to which an expansion of $\gamma$ is then itited. 
Figure 1 - Lines of constant electrical potential $\phi(\alpha, \beta)$ (solid) and of constant conjugate potential $\psi(\alpha, \beta)$ (aashed) for a dipole magnetic field in the equatorial plane, using a simple analytical model of the earth's electric ileld.

Figures 2 - The behavior of magnetic field lines near an X-type (2-a) and an 0-type (2-b) neutral point. In Figure $(2-b)$ the pattern below the neutral point mirrors the one above it and the main axes need not be orthogonal. 


\section{REFERENCES}

Acuna, M.H. and N.F. Ness, The complex magnetic field of Jupiter, submitted to Science, 1975; GSFC x-690-75-42, February 1975.

Akasofu, S.-I. and S. Chapman, The ring current, geomagnetic disturbance, and the Van Alien Belt, J. Geophys. Res. 66, 1321-1350, 1971; correction J. Geophys. Res. 72, 445-446, 1967.

Altschuler, M.D. and G. Newkirk, Jr., Magnetic fields and the structure of the solar corona, Solar Physics 9, 131-149, 1969.

Babcock, H.W., The topology of the Sun's magnetic field and the 22-year cycle, Ap. J. 133, 572-587, 1961.

Backus, G.E., Application of a non-linear boundary value problem for Laplace's equation to gravity and geomagnetic intensity surveys, Quart. J. Mech. Appl. Math. 21, 195-221, 1968.

Backus, G.E., Non-uniqueness of the external geomagnetic field determined by surface intensity measurements, J. Geophys. Res. 75, 6339-6341, 1970.

Backus, G.E., Determination of the external geomagnetic field from intensity measurements, Geophys. Res. Lett., 1, 21, 1974.

Barish, F.D., R. A. Smith and D. P. Stern, Models of the Jovian magnetosphere (abstract), EOS, 55, 694, 1974.

Bartels, J., The eccentric dipole approximating the Earth's magnetic field, Terrestrial Magnetism and Atmospheric Electricity 41, 225-250, 1936.

Baum, P.J., A. Bratenahl and R.S. White, X-ray and electron spectra from the double inverse pinch device, Phys. Fluids 16, 226-230, 1973.

Bonnevier, B., R. Bostrdm and G. Rostocker, A three dimensional model current system for polar magnetic substorms, J. Geophys. Res. 75 , 107-122, 1970. 
Burch, J.L. and R. W. Janetzke, Coordinate transformations for studies of interactions between interplanetary and geomagnetic fields, GSFC $x-621-74-179$, April 1974.

Cain, J.C., S.J. Hendricks, R.A. Langel and V.W. Hudson, A proposed model for the international geomagnetic reference field - 1965. J. Geomagn. Geoelect. 19, 335-355, 1967.

Cain, J.C., Geomagnetic models from satellite surveys, Rev. Geophys. and Space Phys. 9, 259-273, 1971.

Chapman, S., Geomagnetic nomenclature, J. Geophys. Res. 68, 1174, 1963. Chapman, S. and V.C.A. Ferraro, A new theory of magnetic storms, Terrest. Magnetism and Atmospheric Elec. 36, 77-97 and 171-186, 1931. Chapman, S. and J. Bartels, Geomagnetism, Oxford Univ. Press, 1940. Chen, A.J. and D.P. Stern, Adiabatic hamiltonian of charged particle motion in a dipole field, J. Geophys. Res. 80, 690-693, 1975.

Crooker, N.U. and G.L. Siscoe, Model geomagnetic disturbance from asymmetric ring current particles, J. Geophys. Res. 79, 589-603, 1974.

Dungey, J.W., Conditions for the occurence of electrical discharges in astrophysical systems, Phil. Mag., Series 7, 44, 725-738, 1953.

Dungey, J.W., Cosmic Electrodynamics, Cambridge univ. Press, 1958.

Dungey, J.W., The structure of the exosphere, or, adventures in velocity space, in Geophysics: The Earth's Environment, (Proceedings of the 1962 Les Houches Summer School), Gordon and Breach 1963, p. 502-552. 
Elsasser, W.M., Induction effects in terrestrial magnetism, Part I: theoxy, Phys. Rev. 69, 106-116, 1946.

Elsasser, W.M., Induction effects in terrestrial magnetism, Part II: the secular variation, Phys. Rev. 70, 202-212, 1947.

Elsasser, W.M., Induction effects in terrestrial magnetism, Part III: electric modes, Phys. Rev. $\underline{72}, 821-833,1947$.

Elsasser, W.M., Hydrodynamic dynamo theory, Rev. Mod. Phys. 28, 135-163, 1956.

Fairfield, D.H. and G.D. Mead, Magnetospheric mapping with a quantitative geomagnetic field model, J. Geophys. Res. 80, 535-542, 1975.

Forbes, T.G. and T.W. Speiser, Mathematical models of the open magnetosphere: application to dayside auroras, J. Geophys. Res. 78, 7542-7551, 1971 .

Gardner, C., Adiabatic invariants of classical periodic systems, Phys. Rev. 115, 791-794, 1959.

Hansen, W.W., A new type of expansion in radiation problems, Phys. Rev. $47,139-143,1935$.

Hassitt, A., Coordinates in the earth's magnetic field, Publication UCSD-SP-67-4 of the University of California at San Diego, June 1967.

Heppner, J.P. The world magnetic survey, Space Science Reviews 2, 315$354,1963$.

Hilton, H.H., I parameter, a new approximation, J. Geophys. Res. 76, $6952-6954,1971$. 
Hilton, H.H. and M. Schulz, Geomagnetic potential in offset dipole coordinates, J. Geophys. Res. 78, 2324-2330, 1973.

Hones, E.W. Jr., Motions of charged particles trapped in the earth's magnetosphere, J. Geophys. Res. 68, 1209-1219, 1963.

Hurwitz, L. and D.G. Knapp, Inherent vector discrepancies in geomagnetic main field models based on Scalar F, J. Geophys. Res. 79, 30093013,1974

IAGA Commission 2, Working Group 4 International Geomagnetic Reference Field 1965.0, J. Geophys. Res. 74, 4407-4408, 1969.

Kaula, W.M., An Introduction to Planetary Physics, 490 pp., John Wiley and Sons, 1968 .

Kendal1, P.C., S. Chapman, S.-I. Akasofu and P.N. Swartztrauber, The computation of the magnetic field of any axisymmetric current distribution - with magnetospheric applications, Geophys. J. Roy. Astr. Soc. 11, 349-364, 1966.

Kono, M., Uniqueness of the spherical harmonic analysis of the geomagnetic field based on inclination and declination data

Kosik, J.C., Mouvement des particules chargees dans un modele analytique de la magnetosphere de Mead, Annales de Geophysique 27, 11-26, 1971. Kosik, J.C., L'environnement en particules energetiques aux altitudes synchrones au cours des periodes geomagnetiques calmes, Annales de Geophysique 27, 175-180, 1971. 
James, R.W., Transformation of spherical harmonics under change of reference frame, Geophys. J. of Roy. Astr. Soc. 17, 305-316, 1969.

Leighton, R.B., A magneto-kinematic model of the solar cycle, Ap. J. $156,1-26,1969$.

Longmire, C.L., Elementary Plasma Physics, 296 pp., Interscience, New York, 1963.

McDonald, K.L. and R.H. Gunst, An analysis of the eaxth's magnetic field from 1835 to 1965, ESSA technical report IER 46 - IES 1, July 1967. McIlwain, C.E., Coordinates for mapping the distribution of magnetically trapped particles, J. Geophys. Res. 66, 3681-3691, 1961.

McIlwain, C.E., Magnetic coordinates, Radiation Trapped in the Earth's Magnetic Field (B. McCormac Ed.), D. Reidel, 1966; also Space Sci. Rev. $5,585-598,1966$.

Mead, G.D., Deformation of the geomagnetic field by the solar wind, J. Geophys. Res. 69, 1181-1195, 1964.

Mead, G.D. and D.H. Fairfield, A quantitative magnetospheric model derived from spacecraft magnetometer data, J. Geophys. Res. 80, $523-534,1975$.

Midgley, J.E., Perturbation of the geomagnetic field, a spherical harmonic expansion, J. Geophys. Res. 69, 1197-1200, 1964.

Mie, Gustav, Beitrăge zur optik trlber medien, speziell kolloidaler metall8sungen, Ann. Phys. (series 4) 25, 377-445, 1908.

Morse, P.M. and H. Feshbach, Methods of Theoretical Physics, McGraw Hill, 1953. 
Northrop, T.G., The Adiabatic Motion of Charged Particles, $109 \mathrm{pp}$, John Wiley and Sons (Interscience) 1963.

Northrop, T.G. and E. Teller, Stability of the adiabatic motion of charged particles in the earth's field, Phys. Rev. 117, 210-225, 1960.

Olson, W.P., The shape of the tilted magnetopause, J. Geophys. Res. $74,5642-5651,1969$.

Olson, W.P., A model of the distributed magnetospheric currents, J. Geophys. Res. 79, 3731-3738, 1974 .

Olson, W.P. and K.A. Pfitzer, A quantitative model of the magnetospheric magnetic field, J. Geophys. Res. 79, 3739-3748, 1974.

Parker, E.N., Hydrodynamic dynamo models, Ap. J. 122, 293-314, 1955. Parker, E.N., Geomagnetic fluctuations and the form of the outer zone of the Van Allen radiation belt, J. Geophys. Res. 76, 3117-3130, 1960.

Pennington, R.H., Equation of a charged particle shell in a perturbed dipole field, J. Geophys. Res. 66, 709-712, 1961.

Pennington, R.H., Derivation of geomagnetic shell equations, Air Force Weapons Lab. Rept. AFWL-TR-66-152, 1967.

Russbridge, M.G., Non-adiabatic charged particle motion near a magnetic field zero 1ine, Plasma Physics 13, 977-987, 1971.

Russe11, C.T., Geophysical coordinate transformations, Cosmic Electrodynamics 2, 184-196, 1971 .

Schatten, K.H., Current sheet magnetic model for the solar corona, Cosmic Electrodynamics $2,232-245,1971$.

Schatten, K.H. and D.R. Howell, Corona: a program for computing magnetic field lines in the quiet solar corona, GSFC x-692-71-336. August 
Schield, M.A., Pressure balance between solar wind and magnetosphere, J. Geophys. Res. 74, 1275-1286, 1969 (appendix).

Senior, T.B.A., A note on Hansen's vector wave functions, Canad. J. of Phys. 38, 1702-1705, 1960.

Siambis, J.G. and T.G. Northrop, Magnetic field geometry and the adiabatic invariants of particle motion, Phys. Fluids 9, 2001-2005, 1966. Slater, J.C., Quantum Theory of Atomic Structure, vol. 2, appendix 23, McGraw Hill, New York 1960.

Smith, E.J., L. Davis, Jr., D.E. Jones, P.J. Coleman, Jr., D.S. Colburn, P. Dyal, C.P. Sonett and A.M.A. Frandsen, The planetary magnetic field and magnetosphere of Jupiter: Pioneer 10, J. Geophys. Res. 79, 3501-3513, 1974 .

Stacey, F.D., Physics of the Earth, John Wiley and Sons, 1969.

Stern, D.P., The vector potential and motion of charged particles in axisymmetric magnetic fields, J. Geophys. Res. 69, 2715-2719, 1964.

Stern, D.P. Geomagnetic Euler potentials, J. Geophys. Res. 72, 3995$4005,1967$.

Stern, D.P., Euler potentials and geomagnetic drift shells, I. Geophys. Res. $13,4373-4378,1968$.

Stern, D.P., Euler potentials, Amer. J. Phys. 38, 494-501, 1970.

Stern, D.P., Geomagnetic potential in dipole coordinates, J. Geophys. Res. $\underline{76}, 257-258,1971$. 
Stern, D.P., Models of the earth's electric field, GSFC X-602-74-159, May 1974.

Stern, D.P., Charged particle motions in a magnetic field which reduce to motions in a potential, Amer. J. Phys. 43, 1975.

Stern, D.P. and J.H. Bredekamp, Error enhancement in geomagnetic models derived from scalar data, J. Geophys. Res. 80, 1975.

Sweet, P.A., The effect of turbulence on a magnetic field, Monthly Notices Roy. Astr. Soc. 110, 69-83, 1950.

Taylor, H.E. and E.W. Hones, Jr., Adiabatic motion of auroral particles in a model of the electric and magnetic fields surrounding the earth, J. Geophys. Res. 70, 3605-3628, 1965.

Vasyliunas, V.M., Theoretical models of magnetic field line merging, Rev. Geophys. and Space Phys. (to be published, 1975).

Vestine, E.H., The survey of the geomagnetic field in space, Transact. A.G.U. $41,4-21,1960$.

Voigt, G.-H., A 3-dimensional, analytical magnetospheric model with defined magnetopause, z. f. Geophysik 38, 319-346, 1972.

Williams, D.J. and G.D. Mead, Nightside magnetosphere configuration obtained from trapped electrons at 1100 kilometers, J. Geophys. Res. 70, 3017-3029, 1965.

Wilson, G.E., Comparison of three sets of higher order adiabatic plasma equations, Phys. Fluids 13, 1372-1379, 1970.

Zmuda, A.J., The geomagnetic field and its harmonic description, Geomagnetism and Aeronomy 13, 1098-1109, 1973 (in Russian). 


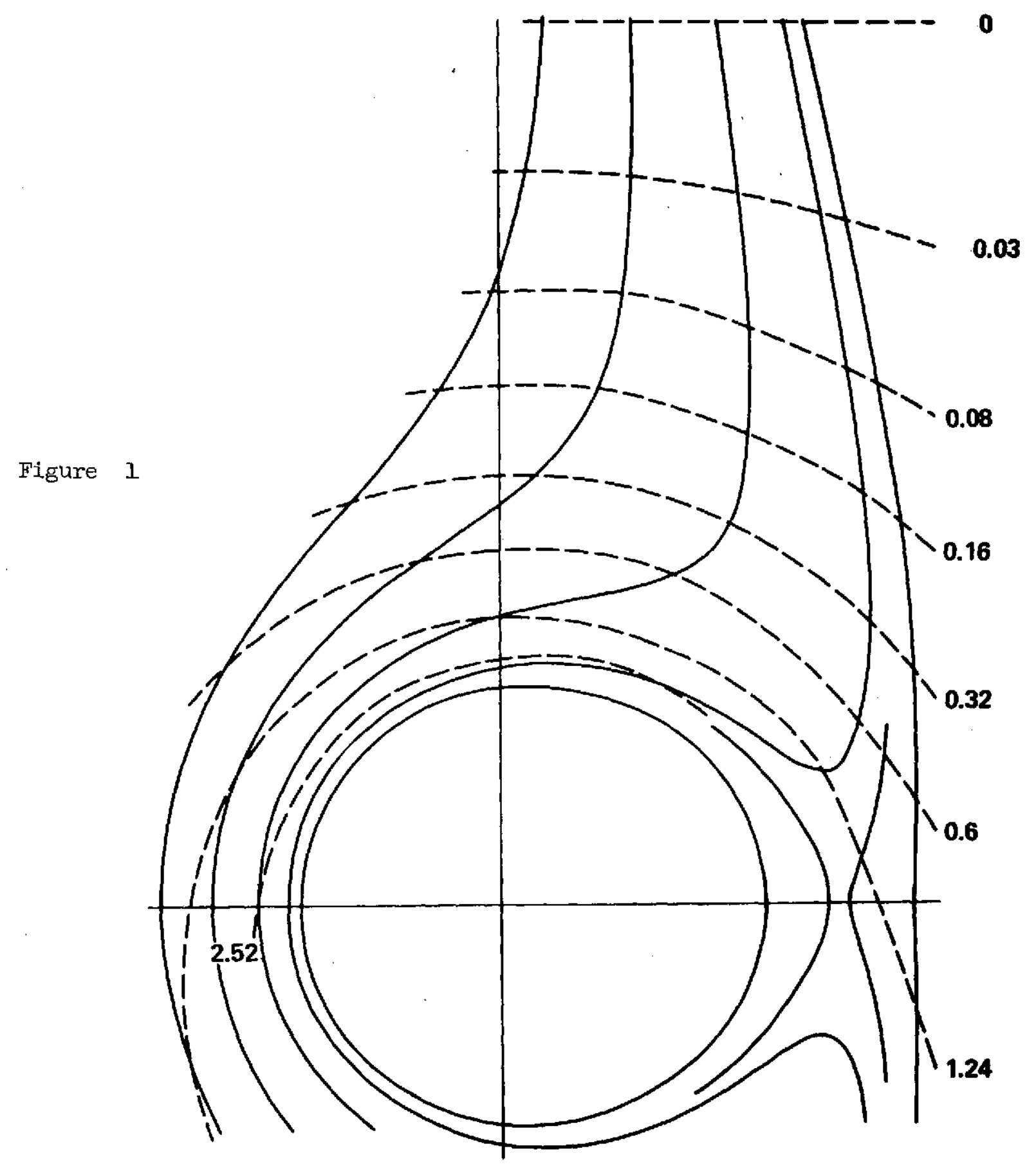



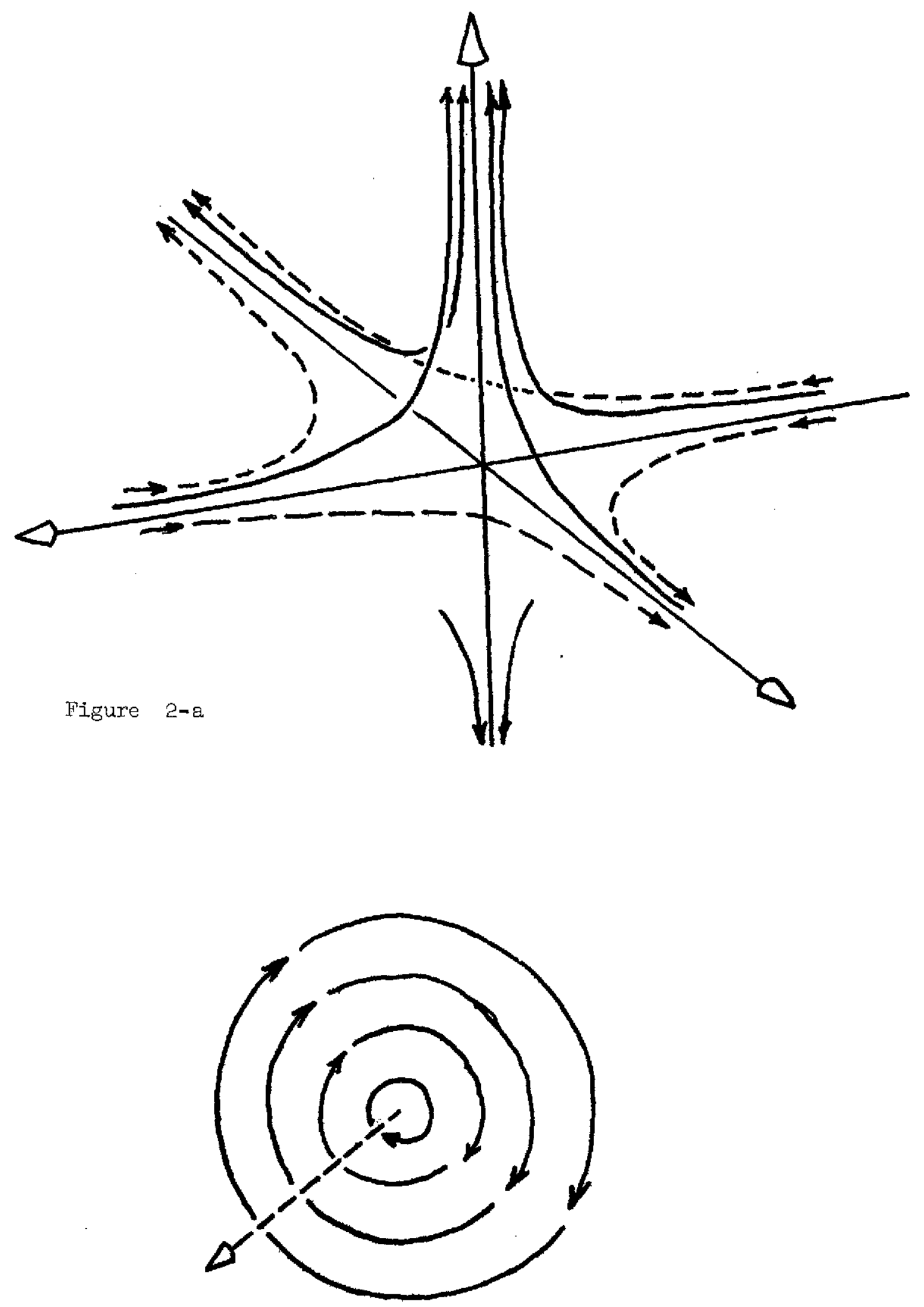

Figure 2-b 